\title{
Lower Bounds for Bruss' Odds Problem with Multiple Stoppings
}

\author{
Tomomi Matsui (Tokyo Institute of Technology) \\ Katsunori Ano (Shibaura Institute of Technology)
}

\begin{abstract}
This paper deals with Bruss' odds problem with multiple stopping chances. A decision maker observes sequentially a sequence of independent $0 / 1$ (failure/success) random variables with the objective to predict the last success correctly with multiple stopping chances. First, we give a nontrivial lower bound of the probability of win (obtaining the last success) for the problem with $m$-stoppings. Next, we show that the asymptotic value for each classical secretary problem with multiple stoppings attains our lower bound. Finally, we prove a conjecture on the classical secretary problem, which gives a connection between the probability of win and the threshold values of the optimal stopping strategy.
\end{abstract}

\section{Introduction.}

We discuss asymptotic lower bounds of probability of "win" (i.e., obtaining the last success) for odds problem with multiple stoppings, which has some general setting in optimal stopping theory. The problem may be stated as follows.

Suppose that you know that you will be given a random sequence of zeros and ones; you know how long the sequence will be, say $N$; you know that you will be given the digits one by one; you do not know what the next digit will be zero or one, but you do know the probability that it will be a one. You are allowed to claim at most $m$ times, when you see a one, "the one that I observe now, will be the last one in the sequence." You will win if your last claim turns out to be indeed the last one.

The above problem is called an $m$-stopping odds problem. You want to win with maximal probability. Of course, we assume $m<<N$, for instance $m=1$, which is the Bruss' optimal stopping problem, and which is closely related to the classical secretary problem.

Now we define a class of feasible policies, say $\Pi$. Each policy in $\Pi$ gets input of a sequence of $0 / 1$ random variables $\mathcal{X}=\left(X_{1}, X_{2}, \ldots, X_{N}\right)$, that is, Bernoulli sequence. We say "success" if $X_{i}=1$ and "failure" if $X_{i}=0$. A policy $\pi \in \Pi$ 
returns (indices of) variables in $\mathcal{X}$ at which you claim to observe the last success. The value of the policy, which is called the probability of win, is its probability to win: $\mathbb{P}[\pi(\mathcal{X})=\infty]$ (where "=1" means "includes the variable of the last success"). An optimal policy would be $\pi^{*}=\arg \max _{\pi \in \Pi} \mathbb{P}[\pi(\mathcal{X})=\infty]$.

This is an attractive problem setting. We may quote from Bruss Bruss (2000): "Many stopping problems are of a similar kind. One often wants to stop on the very last success. For instance, investors are typically interested in stopping on the last success in a given period, where a success is a price increase in a long position and a decrease in a short position. Similarly, venture capital investors often try to put all reserved capital in the last technological innovation in the targeted field. In secretary problems, we want to select the best candidate (which means stopping on the last record value) and so on."

The single stopping problem has an elegant and a simple optimal stopping strategy determined by Odds theorem or Sum the Odds theorem. A typical lower bound for an asymptotic optimal value (the probability of win), when $N$ goes to infinity, is shown to be $e^{-1}$ in Bruss Bruss $(2000,2003)$. The value often appears in the literature of the many modifications of the secretary problem having a specified probability of success, $\mathbb{P}\left[X_{i}=1\right]=1 / i$ (see, e.g., Pfeifer Pfeifer (1989), Samuels Samuels (1992) for a review and others), and in the one of the variations of prophet inequality based on relative ranks (see, e.g., Hill and Krengel Hill and Krengel (1992) and Hill and Kennedy Hill and Kennedy (1992)). The value $e^{-1}$ also appears in the asymptotic threshold value of the optimal stopping strategy for the secretary problem. For large $N$, the optimal strategy (for the secretary problem) is to pass all the candidates until $e^{-1} N$ and then stop at the first relative best (if any) thereafter. Other variations of the single stopping problem are studied by Bruss and Paindaveine Bruss and Paindaveine (2000) for stopping on the $\ell$-th last success, Hsiau and Yang Hsiau and Yang (2002) for Markov-dependent trials, and Tamaki Tamaki (2010) for stopping on any of the last $\ell$ successes.

For the multiple stopping odds problem, Ano, Kakinuma and Miyoshi Ano et al. (2010) provided an optimal strategy based on a (multiple) threshold strategy. They also showed that the double stopping odds problem has a lower bound $e^{-\frac{3}{2}}+e^{-1}$ for an asymptotic optimal value (the probability of win) when $N$ goes to infinity. It is interesting that their lower bound is equal to the asymptotic optimal value of the double stopping secretary problem. Another variation of Markov-dependent trials with multiple stoppings is studied by Ano, Kakie and Miyoshi Ano et al. (2011).

For odds problems and the secretary problems with multiple stoppings, this paper answers to the following questions.

(Q1) What is the maximum probability of win and the lower bound of the odds problem (and not only for the secretary problem) with $m$-stoppings?

We give an explicit formula of the probability of win for the $m$-stopping odds problem, which is based on an enumeration technique for specified integer sequences.

(Q2) What is the asymptotic lower bound of the probability of win for the 
$m$-stopping odds problem, when $N$ goes to infinity?

For any fixed number $m$ of stopping chances, we give an asymptotic lower bound of the probability of win for odds problem.

(Q3) Is the greatest lower bound of the $m$-stopping odds problem equal to the asymptotic optimal value (the probability of win) of the secretary problem with $m$-stopping chances? In other words, does the secretary problem still keep benchmark position of the bound for odds problem?

In the secretary problem, multiple stopping setting may go back to Gilbert and Mosteller Gilbert and Mosteller (1966). Asymptotic optimal values of secretary problems for $m=1,2,3$ and 4 appears in Gilbert and Mosteller (1966); Bruss (1988). The single stopping odds problem has a lower bound $e^{-1}$, shown by Bruss Bruss (2003), which is equal to the asymptotic optimal value of the classical secretary problem. For double stopping case $(m=2)$, Ano, Kakinuma and Miyoshi Ano et al. (2010) derived a lower bound $e^{-\frac{3}{2}}+e^{-1}$ of odds problem, which is equal to the asymptotic optimal value of the double stopping secretary problem.

We prove that the above property holds for any $m$, which also implies the tightness of our lower bounds. We also give asymptotic optimal values of the secretary problem with $m$ stopping chances for $m=5,6,7,8,9$ and 10 in Table 1 .

(Q4) Is the lower bound of the secretary problem remarkably composed of the asymptotic threshold values in the optimal multiple stopping strategy?

For example, it is known that the triple of threshold values $\left(i_{N}^{(3)}, i_{N}^{(2)}, i_{N}^{(1)}\right)$ for the secretary problem with three stopping chances satisfies

$$
\lim _{N \rightarrow \infty}\left(\frac{i_{N}^{(3)}}{N}, \frac{i_{N}^{(2)}}{N}, \frac{i_{N}^{(1)}}{N}\right)=\left(e^{-\frac{47}{24}}, e^{-\frac{3}{2}}, e^{-1}\right)
$$

and the corresponding probability of win converges to $\left(e^{-\frac{47}{24}}+e^{-\frac{3}{2}}+e^{-1}\right)$. It is also known that a similar relation holds when the number of stopping chances is $1,2,3$, or 4 . We prove a conjecture on the secretary problem indicated by Gilbert and Mosteller Gilbert and Mosteller (1966) and raised explicitly in Ano et al. (2010), which shows a beautiful connection between threshold values of the optimal stopping strategy and the probability of win. Let $\left(i_{N}^{(m)}, i_{N}^{(m-1)}\right.$, $\left.\ldots, i_{N}^{(1)}\right)$ be a vector of optimal threshold values for the secretary problem with $m$-stopping chances, where $N$ denotes the number of random variables. We prove the following equality

$$
\lim _{N \rightarrow \infty}\left(\frac{i_{N}^{(m)}}{N}+\frac{i_{N}^{(m-1)}}{N}+\cdots+\frac{i_{N}^{(1)}}{N}\right)=\lim _{N \rightarrow \infty} \mathbb{P}_{N}^{(\text {win })}(m)
$$

where $\mathbb{P}_{N}^{(\text {win })}(m)$ denotes the corresponding probability of win for the secretary problem with $m$-stopping chances. 


\section{Threshold Strategy and Probability of Win.}

Let $\mathcal{X}=\left(X_{1}, X_{2}, \ldots, X_{N}\right)$ be a given Bernoulli sequence, i.e., a sequence of independent $0 / 1$ random variables. For any $i \in\{1,2, \ldots, N\}$, we denote $p_{i}=$ $\mathbb{E}\left[X_{i}\right]$. Throughout this paper, we assume that $0<p_{i}<1$ for any $i$. We denote a probability of failure $1-p_{i}$ by $q_{i}$ and an odds $\frac{p_{i}}{q_{i}}$ of $X_{i}$ by $r_{i}$, for each $i \in\{1,2, \ldots, N\}$. In this section, we discuss an optimal strategy for the $m$-stopping odds problem defined on $\mathcal{X}$.

By applying dynamic programming (DP) techniques, Ano, Kakinuma and Miyoshi Ano et al. (2010) showed that an optimal policy has the structure of a multiple threshold strategy, denoted by Threshold $\left(i^{(m)}, i^{(m-1)}, \ldots, i^{(1)}\right)$, defined by threshold values satisfying $1 \leq i^{(m)} \leq i^{(m-1)} \leq \cdots \leq i^{(1)} \leq N$. The threshold strategy Threshold $\left(i^{(m)}, i^{(m-1)}, \ldots, i^{(1)}\right)$ selects a variable of success (at which you claim to observe the last success) if and only if the number of previously selected variables is less than the number of passed threshold values on and before the observation. For example, we consider a case that $\left(X_{1}, X_{2}, \ldots, X_{8}\right)$ has a vector of the realized values $(0,1,1,0,0,1,1,1)$. If we apply the threshold strategy Threshold $(2,4,5)$, for the 3 -stopping odds problem, then the strategy selects variables $X_{2}, X_{6}, X_{7}$, which does not includes the last success $X_{8}$. More precisely, the threshold strategy Threshold $\left(i^{(m)}, i^{(m-1)}, \ldots, i^{(1)}\right)$ selects a set of variables indexed by a set of indices $\pi(1)$ defined by the following recurrence relation

$$
\begin{aligned}
\pi(m+1)=\emptyset \\
\pi(k)=\pi(k+1) \cup\left\{\min \left\{\begin{array}{l|l}
i \in\{1,2, \ldots, N\} & \begin{array}{l}
i^{(k)} \leq i, X_{i}=1, \\
i^{\prime}<i\left(\forall i^{\prime} \in \pi(k+1)\right) .
\end{array}
\end{array}\right\}(\forall k \in\{m, m-1, \ldots, 1\}),\right.
\end{aligned}
$$

where we define $\{\min \{\emptyset\}\}=\emptyset$.

First, we discuss the probability of win of Threshold $\left(i^{(m)}, i^{(m-1)}, \ldots, i^{(1)}\right)$. We introduce a partition $\left\{B_{m+1}, B_{m}, \ldots, B_{1}\right\}$ of index set $\{1,2, \ldots, N\}$ defined by

$$
B_{k}= \begin{cases}\left\{i \in\{1,2, \ldots, N\} \mid i^{(1)} \leq i \leq N\right\} & (k=1), \\ \left\{i \in\{1,2, \ldots, N\} \mid i^{(k)} \leq i<i^{(k-1)}\right\} & (1<k \leq m), \\ \left\{i \in\{1,2, \ldots, N\} \mid 1 \leq i<i^{(m)}\right\} & (k=m+1) .\end{cases}
$$

Each index set in $\left\{B_{m+1}, B_{m}, \ldots, B_{1}\right\}$ is called a block. We identify the set of indices in each block with the set of corresponding variables, if there is no ambiguity. Given a $0-1$ vector $\boldsymbol{x} \in\{0,1\}^{N}$, we introduce a pattern vector of $\boldsymbol{x}$, denoted by $\boldsymbol{b}(\boldsymbol{x})=\left(b_{m}, b_{m-1}, \ldots, b_{1}\right)$, satisfying that $b_{k}$ is the number of $1 \mathrm{~s}$ in the subvector of $\boldsymbol{x}$ defined by block $B_{k}$, i.e., $b_{k}=\sum_{i \in B_{k}} x_{i}(k \in\{1,2, \ldots, m\})$. Here we note that elements of vector $\boldsymbol{b}(\boldsymbol{x})$ are arranged in decreasing order of indices and block $B_{m+1}$ is ignored. For any vector $\boldsymbol{b}=\left(b_{d}, b_{d-1}, \ldots, b_{1}\right)$, we say that a vector $\left(b_{d^{\prime}}, b_{d^{\prime}-1}, \ldots, b_{1}\right)$ satisfying $d \geq d^{\prime} \geq 1$ is a left truncated subvector of $\boldsymbol{b}$.

If an index corresponding to the last success is obtained by executing Threshold $(\boldsymbol{i})$, we say that a vector of realized values $\left(x_{1}, x_{2}, \ldots, x_{N}\right) \in\{0,1\}^{N}$ of $\left(X_{1}, X_{2}, \ldots, X_{N}\right)$ 
is winning. When we consider a single-stopping odds problem (discussed in Bruss (2003)), a vector $\boldsymbol{x} \in\{0,1\}^{N}$ is winning if and only if its pattern vector $\boldsymbol{b}(\boldsymbol{x})$ satisfies $b_{1}=1$, i.e., one-dimensional vector $(1) \in\{0,1\}^{1}$ is a left truncated subvector of $\boldsymbol{b}(\boldsymbol{x})$. The probability of win of $\operatorname{Threshold}\left(i^{(1)}\right)$ is equal to $\left(\prod_{i \in B_{1}} q_{i}\right) \cdot\left(\sum_{i \in B_{1}} r_{i}\right)$.

Next, we consider the case that $m=2$ (discussed in Ano et al. (2010)). We assume that $\left|B_{1}\right| \geq 2$. It is easy to show that a vector $\boldsymbol{x} \in\{0,1\}^{N}$ is winning if and only if its pattern vector $\boldsymbol{b}(\boldsymbol{x})$ has a left truncated subvector in $\{(1),(1,0),(0,2)\}$. Thus, the probability of win of $\operatorname{Threshold}\left(i^{(2)}, i^{(1)}\right)$ is equal to

$$
\left(\prod_{i \in B_{1}} q_{i}\right)\left(\sum_{i \in B_{1}} r_{i}\right)+\left(\prod_{i \in B_{2} \cup B_{1}} q_{i}\right)\left(\sum_{i \in B_{2}} r_{i}+\sum_{\left\{i, i^{\prime}\right\} \subseteq B_{1}} r_{i} r_{i^{\prime}}\right) .
$$

When $m \leq 5$, a brute force method shows that a vector $\boldsymbol{x} \in\{0,1\}^{N}$ is winning if and only if there exists a set $\Xi_{k}(k \in\{1,2, \ldots, 5\})$ that includes a left truncated subvector of $\boldsymbol{b}(\boldsymbol{x})$, where

$$
\begin{aligned}
& \Xi_{1}=\{(1)\}, \\
& \Xi_{2}=\{(1,0),(0,2)\} \\
& \Xi_{3}=\{(1,0,0),(0,2,0),(0,1,2),(0,0,3)\}, \\
& \Xi_{4}=\left\{\begin{array}{l}
(1,0,0,0),(0,2,0,0),(0,1,2,0),(0,1,1,2),(0,1,0,3), \\
(0,0,3,0),(0,0,2,2),(0,0,1,3),(0,0,0,4)
\end{array}\right\}, \\
& \Xi_{5}=\left\{\begin{array}{l}
(1,0,0,0,0)(0,2,0,0,0)(0,1,2,0,0)(0,1,1,2,0)(0,1,1,1,2)(0,1,1,0,3) \\
(0,1,0,3,0)(0,1,0,2,2)(0,1,0,1,3)(0,1,0,0,4)(0,0,3,0,0)(0,0,2,2,0) \\
(0,0,2,1,2)(0,0,2,0,3)(0,0,1,3,0)(0,0,1,2,2)(0,0,1,1,3)(0,0,1,0,4) \\
(0,0,0,4,0)(0,0,0,3,2)(0,0,0,2,3)(0,0,0,1,4)(0,0,0,0,5)
\end{array}\right.
\end{aligned}
$$

The following table of the sizes of $\Xi_{k}$ is obtained by a naive computer program for enumeration.

\begin{tabular}{c||l|l|l|l|r|r|r|r|r|r|r|l}
$k$ & 1 & 2 & 3 & 4 & 5 & 6 & 7 & 8 & 9 & 10 & 11 & $\cdots$ \\
\hline$\left|\Xi_{k}\right|$ & 1 & 2 & 4 & 9 & 23 & 65 & 197 & 626 & 2056 & 6918 & 23714 & $\cdots$
\end{tabular}

Here we discuss an example of the 5-stopping odds problem. Assume that $\boldsymbol{x} \in\{0,1\}^{N}$ has a pattern vector $\boldsymbol{b}(\boldsymbol{x})=(3,1,0,2,0)$. The threshold strategy selects first variable of success in block $B_{5}$, rejects second and third variables of success in $B_{5}$, and selects all the remained three variables of success in $B_{4} \cup B_{2}$. The vector $\boldsymbol{x}$ is winning, since $\boldsymbol{b}(\boldsymbol{x})=(3,1,0,2,0)$ has a left-truncated subvector $(0,2,0) \in \Xi_{3}$.

Now we discuss the general case. First, we show a necessary and sufficient condition that a vector $\boldsymbol{x} \in\{0,1\}^{N}$ becomes a winning vector of the $m$-stopping odds problem. Throughout this paper, $\mathbb{Z}_{+}$denotes the set of non-negative 
integers. We define a set of $k$-vectors

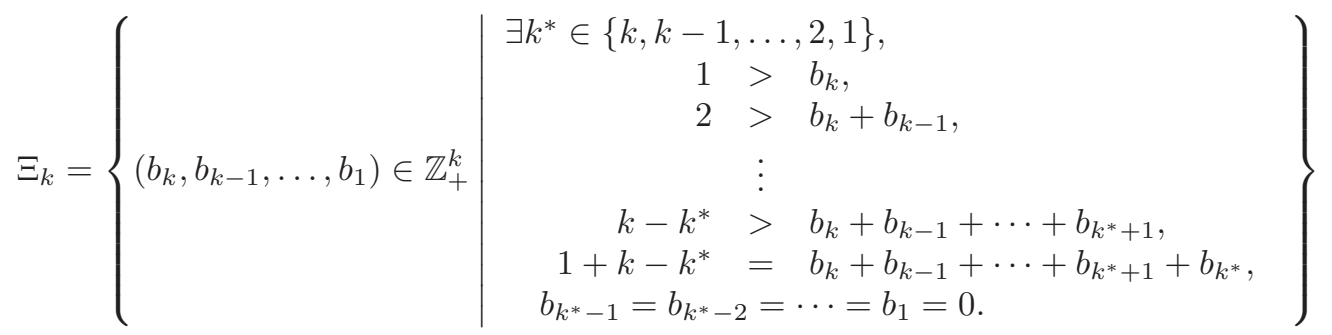

for each positive integer $k$. Here we note that when $k^{*}=k$ in the above definition, all the inequalities are ignored and $(1,0,0, \ldots, 0) \in \mathbb{Z}_{+}^{k}$ becomes a unique vector satisfying the remaining conditions. Thus, the set $\Xi_{k}$ contains the $k$-vector $(1,0,0, \ldots, 0)$.

Lemma 2.1. Let $\boldsymbol{x} \in\{0,1\}^{N}$ be a vector satisfying that $\exists k \in\{1,2, \ldots, m\}$, $\exists \boldsymbol{b} \in \Xi_{k}, \boldsymbol{b}$ is a left truncated subvector of $\boldsymbol{b}(\boldsymbol{x})$. Then $\boldsymbol{x}$ is a winning vector of the m-stopping odds problem.

Proof. Let $k^{*}$ be an index of variable appearing in the definition of $\Xi_{k}$ with respect to $\boldsymbol{b} \in \Xi_{k}$. The definition of $\Xi_{k}$ implies that block $B_{k^{*}}$ includes the index of the variable of the last success and the number of successes with respect to $\boldsymbol{x}$ in blocks $B_{k} \cup B_{k-1} \cup \cdots \cup B_{k^{*}}$ is equal to $1+k-k^{*}$.

When we apply the threshold strategy, the number of selected variables in blocks $B_{m} \cup B_{m-1} \cup \cdots \cup B_{k+1}$ is less than or equal to $m-k$, obviously.

Thus, when we observe the variables of the last success, the number of previously selected variables is less than or equal to $(m-k)+\left(1+k-k^{*}\right)-1=$ $m-k^{*}$, which is less than the number of passed threshold values $m-k^{*}+1$, where passed threshold values are $\left\{i^{(m)}, \ldots, i^{\left(k^{*}\right)}\right\}$.

It implies that the threshold strategy selects the variable of the last success and thus $\boldsymbol{x}$ is winning. (Here we note that all the variables of success in $B_{k} \cup$ $B_{k-1} \cup \cdots \cup B_{k^{*}}$ are also selected.)

Next, we discuss the inverse implication.

Lemma 2.2. For any winning vector $\boldsymbol{x} \in\{0,1\}^{N}$ of the m-stopping odds problem, there exists an index $k \in\{1,2, \ldots, m\}$ and a vector $\boldsymbol{b} \in \Xi_{k}$ satisfying that $\boldsymbol{b}$ is a left truncated subvector of $\boldsymbol{b}(\boldsymbol{x})$.

Proof. We show the above by induction on $m$. When $m=1$, it is obvious. We assume that the above property holds for each integer in $\{1,2, \ldots, m-1\}$. (i) Consider a case that when we apply the threshold strategy Threshold $\left(i^{(m)}, \ldots, i^{(1)}\right)$, the number of previously selected variables is strictly less than the number of passed threshold values at every time instance just after a variable is selected. Then, it is easy to see that even if we apply the threshold strategy Threshold $\left(i^{(m-1)}, \ldots, i^{(1)}\right)$ to $\boldsymbol{x}$, the set of selected variables remains unchanged, and thus $\boldsymbol{x}$ is also a winning vector of the $(m-1)$-stopping odds problem. The induction hypothesis implies that, $\exists k \in\{1,2, \ldots, m-1\}, \exists \boldsymbol{b} \in \Xi_{k}$ satisfying that $\boldsymbol{b}$ is a left truncated subvector of $\boldsymbol{b}(\boldsymbol{x})$. 
(ii) Next, we consider the remaining case. Let $i^{*} \in\{1,2, \ldots, N\}$ be the minimum index of a variable selected by the threshold strategy $\operatorname{Threshold}\left(i^{(m)}, \ldots, i^{(1)}\right)$ under the condition that just after $x_{i^{*}}$ is selected, the number of previously selected variables becomes equal to the number of passed threshold values. We denote the block including $i^{*}$ by $B_{k^{*}}$. From the minimality of $i^{*}, m$-vector $\boldsymbol{b}(\boldsymbol{x})=\left(b_{m}, b_{m-1}, \ldots, b_{1}\right)$ satisfies inequalities

$$
\begin{aligned}
1 & >b_{m}, \\
2 & >b_{m}+b_{m-1}, \\
& \vdots \\
m-k^{*} & >b_{m}+b_{m-1}+\cdots+b_{k^{*}+1} .
\end{aligned}
$$

Here we note that when $k^{*}=m$, we omit the above inequalities. Since the number of previously selected variables is equal to the number of passed threshold values just after $x_{i^{*}}$ is selected, all the variables in $\left\{X_{i} \mid i \in B_{k^{*}}\right.$ and $\left.i^{*}<i\right\}$ are not selected and thus we have the equality

$$
1+m-k^{*}=b_{m}+b_{m-1}+\cdots+b_{k^{*}+1}+b_{k^{*}} .
$$

(ii-a) If $x_{i^{*}}$ is the last success, then $b_{k^{*}-1}=b_{k^{*}-2}=\cdots=b_{1}=0$, which yields $\boldsymbol{b}(\boldsymbol{x}) \in \Xi_{m}$.

(ii-b) Lastly, we consider the case that $x_{i^{*}}$ is not the last success. Clearly, $B_{k^{*}}$ does not include the index of the variable of the last success, since variables in $\left\{x_{i} \mid i \in B_{k^{*}}\right.$ and $\left.i^{*}<i\right\}$ are not selected.

The equality (6) implies that if we apply Threshold $\left(i^{\left(k^{*}-1\right)}, \ldots, i^{(1)}\right)$ to $\boldsymbol{x}$, the set of selected variables in $B_{k^{*}-1} \cup \cdots \cup B_{2} \cup B_{1}$ remains unchanged and thus $\boldsymbol{x}$ is a winning vector of the $\left(k^{*}-1\right)$-stopping odds problem. The induction hypothesis implies the desired result.

We can also show the following uniqueness.

Corollary 2.3. For any winning vector $\boldsymbol{x} \in\{0,1\}^{N}$ of the m-stopping odds problem, there exists a unique index $k \in\{1,2, \ldots, m\}$ and a unique vector $\boldsymbol{b} \in \Xi_{k}$ satisfying that $\boldsymbol{b}$ is a left truncated subvector of $\boldsymbol{b}(\boldsymbol{x})$.

Proof. The definition of $\Xi_{k}$ directly implies that for any pair of vectors $\boldsymbol{b}^{\prime} \in \Xi_{k^{\prime}}$ and $\boldsymbol{b}^{\prime \prime} \in \Xi_{k^{\prime \prime}}$, when $\boldsymbol{b}^{\prime \prime}$ is a left truncated subvector of $\boldsymbol{b}^{\prime}$, then both $\boldsymbol{b}^{\prime}=\boldsymbol{b}^{\prime \prime}$ and $k^{\prime}=k^{\prime \prime}$ hold. As a consequence, we have the desired result.

Summarizing the above properties, we have the following theorem.

Theorem 2.4. A vector $\boldsymbol{x} \in\{0,1\}^{N}$ is a winning vector of the $m$-stopping odds problem if and only if there exists a unique integer $k \in\{1,2, \ldots, m\}$ satisfying that a pattern vector $\boldsymbol{b}(\boldsymbol{x})=\left(b_{m}, b_{m-1}, \ldots, b_{1}\right)$ has a left truncated subvector $\left(b_{k}, b_{k-1}, \ldots, b_{1}\right) \in \Xi_{k}$.

Next, we discuss the probability of win. Given an index subset $B \subseteq\{1,2, \ldots, N\}$ 
and a positive integer $b$, we define

$$
f^{b}(B)=\left\{\begin{array}{cc}
\sum_{B^{\prime} \subseteq B,\left|B^{\prime}\right|=b}\left(\prod_{i \in B^{\prime}} r_{i}\right) & (|B| \geq b), \\
0 & (|B|<b)
\end{array}\right.
$$

which is called an elementary symmetric polynomial defined on $\left\{r_{i} \mid i \in B\right\}$. We define $f^{0}(B)=1$.

Theorem 2.5. Given a threshold strategy Threshold $\left(i^{(m)}, \ldots, i^{(2)}, i^{(1)}\right)$ for the $m$-stopping odds problem defined on $X_{1}, X_{2}, \ldots, X_{N}$, the corresponding probability of win is equal to

$$
\sum_{k=1}^{m}\left(\left(\prod_{i \in B_{k} \cup \cdots \cup B_{2} \cup B_{1}} q_{i} \sum_{\left(b_{k}, \ldots, b_{1}\right) \in \Xi_{k}}\left(f^{b_{k}}\left(B_{k}\right) f^{b_{k-1}}\left(B_{k-1}\right) \cdots f^{b_{1}}\left(B_{1}\right)\right)\right) .\right.
$$
$\mathbb{Z}_{+}^{k}$,

Proof. The definition of $f^{b}(B)$ directly implies that for any $\left(b_{k}, b_{k-1}, \ldots, b_{1}\right) \in$

$$
\begin{aligned}
\mathbb{P}\left[\bigcap_{k^{\prime}=1}^{k}\left\{\sum_{i \in B_{k^{\prime}}} X_{i}=b_{k^{\prime}}\right\}\right] & =\left(\left(\prod_{i \in B_{k}} q_{i}\right) f^{b_{k}}\left(B_{k}\right)\right) \cdots\left(\left(\prod_{i \in B_{1}} q_{i}\right) f^{b_{1}}\left(B_{1}\right)\right) \\
& =\left(\prod_{i \in B_{k} \cup \cdots \cup B_{2} \cup B_{1}} q_{i}\right)\left(f^{b_{k}}\left(B_{k}\right) f^{b_{k-1}}\left(B_{k-1}\right) \cdots f^{b_{1}}\left(B_{1}\right)\right) .
\end{aligned}
$$

From the uniqueness appearing in Theorem 2.4, the probability of win of the threshold strategy Threshold $\left(i^{(m)}, \ldots, i^{(2)}, i^{(1)}\right)$ is equal to

$$
\begin{aligned}
& \sum_{k=1}^{m} \sum_{\left(b_{k}, \ldots, b_{1}\right) \in \Xi_{k}} \mathbb{P}\left[\bigcap_{k^{\prime}=1}^{k}\left\{\sum_{i \in B_{k^{\prime}}} X_{i}=b_{k^{\prime}}\right\}\right] \\
& \quad=\sum_{k=1}^{m}\left(\left(\prod_{i \in B_{k} \cup \cdots \cup B_{2} \cup B_{1}} q_{i}\right) \sum_{\left(b_{k}, \ldots, b_{1}\right) \in \Xi_{k}}\left(f^{b_{k}}\left(B_{k}\right) f^{b_{k-1}}\left(B_{k-1}\right) \cdots f^{b_{1}}\left(B_{1}\right)\right)\right) .
\end{aligned}
$$

For example, when $m=3$, the set $\Xi_{3}$ of winning patterns includes four vectors $\Xi_{3}=\{(1,0,0),(0,2,0),(0,1,2),(0,0,3)\}$ and thus the probability of win is equal to

$$
\begin{aligned}
& \left(\prod_{i \in B_{1}} q_{i}\right)\left(\sum_{i \in B_{1}} r_{i}\right)+\left(\prod_{i \in B_{2} \cup B_{1}} q_{i}\right)\left(\sum_{i \in B_{2}} r_{i}+\sum_{\left\{i_{1}, i_{2}\right\} \subseteq B_{1}} r_{i_{1}} r_{i_{2}}\right) \\
& +\left(\prod_{i \in B_{3} \cup B_{2} \cup B_{1}} q_{i}\right)\left(\begin{array}{l}
\sum_{i \in B_{3}} r_{i}+\sum_{\left\{i_{1}, i_{2}\right\} \subseteq B_{2}} r_{i_{1}} r_{i_{2}} \\
\left.+\left(\sum_{i \in B_{2}} r_{i}\right)\left(\sum_{\left\{i_{1}, i_{2}\right\} \subseteq B_{1}} r_{i_{1}} r_{i_{2}}\right)+\sum_{\left\{i_{1}, i_{2}, i_{3}\right\} \subseteq B_{1}} r_{i_{1}} r_{i_{2}} r_{i_{3}}\right)
\end{array}\right) .
\end{aligned}
$$




\section{Lower Bounds.}

In this section, we discuss a lower bound of the probability of win.

First, we discuss a solution vector $\left(\lambda_{1}, \lambda_{2}, \ldots, \lambda_{m}\right)$ of an equality system;

$$
\sum_{\left(b_{k}, \ldots, b_{1}\right) \in \Xi_{k}}\left(\frac{\lambda_{k}^{b_{k}}}{b_{k} !} \frac{\lambda_{k-1}^{b_{k-1}}}{b_{k-1} !} \cdots \frac{\lambda_{1}^{b_{1}}}{b_{1} !}\right)=1 \quad(k \in\{1,2, \ldots, m\})
$$

which plays an important role in this paper. Let us begin with small examples. The description of $\Xi_{1}, \Xi_{2}, \Xi_{3}$ and $\Xi_{4}$ (see (1)-(4)) implies that $\left(\lambda_{1}, \lambda_{2}, \lambda_{3}, \lambda_{4}\right)$ is a solution of the following system;

$$
\begin{array}{r}
\frac{\lambda_{1}^{1}}{1 !}=1, \\
\frac{\lambda_{2}^{1} \lambda_{1}^{0}}{1 ! 0 !}+\frac{\lambda_{2}^{0} \lambda_{1}^{2}}{0 ! 2 !}=1, \\
\frac{\lambda_{3}^{1} \lambda_{2}^{0} \lambda_{1}^{0}}{1 ! 0 ! 0 !}+\frac{\lambda_{3}^{0} \lambda_{2}^{2} \lambda_{1}^{0}}{0 ! 2 ! 0 !}+\frac{\lambda_{3}^{0} \lambda_{2}^{1} \lambda_{1}^{2}}{0 ! 1 ! 2 !}+\frac{\lambda_{3}^{0} \lambda_{2}^{0} \lambda_{1}^{3}}{0 ! 0 ! 3 !}=1, \\
\frac{\lambda_{4}^{1} \lambda_{3}^{0} \lambda_{2}^{0} \lambda_{1}^{0}}{1 ! 0 ! 0 ! 0 !}+\frac{\lambda_{4}^{0} \lambda_{3}^{2} \lambda_{2}^{0} \lambda_{1}^{0}}{0 ! 2 ! 0 ! 0 !}+\frac{\lambda_{4}^{0} \lambda_{3}^{1} \lambda_{2}^{2} \lambda_{1}^{0}}{0 ! 1 ! 2 ! 0 !}+\frac{\lambda_{4}^{0} \lambda_{3}^{1} \lambda_{2}^{1} \lambda_{1}^{2}}{0 ! 1 ! 1 ! 2 !}+\frac{\lambda_{4}^{0} \lambda_{3}^{1} \lambda_{2}^{0} \lambda_{1}^{3}}{0 ! 1 ! 0 ! 3 !} \\
+\frac{\lambda_{4}^{0} \lambda_{3}^{0} \lambda_{2}^{3} \lambda_{1}^{0}}{0 ! 0 ! 3 ! 0 !}+\frac{\lambda_{4}^{0} \lambda_{3}^{0} \lambda_{2}^{2} \lambda_{1}^{2}}{0 ! 0 ! 2 ! 2 !}+\frac{\lambda_{4}^{0} \lambda_{3}^{0} \lambda_{2}^{1} \lambda_{1}^{3}}{0 ! 0 ! 1 ! 3 !}+\frac{\lambda_{4}^{0} \lambda_{3}^{0} \lambda_{2}^{0} \lambda_{1}^{4}}{0 ! 0 ! 0 ! 4 !}=1 .
\end{array}
$$

The above system has a solution $\left(\lambda_{1}, \lambda_{2}, \lambda_{3}, \lambda_{4}\right)=(1,1 / 2,11 / 24,505 / 1152)$. (Our theorem appearing below shows that $e^{-1}+e^{-3 / 2}+e^{-47 / 24}+e^{-2761 / 1152}$ gives a lower bound of the probability of win.) Here we note that $\lambda_{5}=$ 209519/491520 and $\lambda_{6}=49081919440723 / 117413668454400$. Values $\lambda_{7}, \ldots, \lambda_{10}$ appear in our mimeo Matsui and Ano (2012). First, we show the uniqueness of a solution. The following property is discussed by Gilbert and Mosteller Gilbert and Mosteller (1966) in a setting of the secretary problem.

Lemma 3.1. For any $k \in\{1,2, \ldots, m\}, \Xi_{k}$ includes the unit $k$-vector $\boldsymbol{e}=$ $(1,0,0, \ldots, 0)$ and every vector $\left(b_{k}, b_{k-1}, \ldots, b_{1}\right) \in \Xi_{k} \backslash\{\boldsymbol{e}\}$ satisfies $b_{k}=0$.

Proof. It is obvious that $\boldsymbol{e} \in \Xi_{k}$. Conversely, if a vector $\boldsymbol{b}=\left(b_{k}, \ldots, b_{1}\right) \in$ $\Xi_{k}$ satisfies $b_{k} \neq 0$, then the index $k^{*}$ appearing in the definition of $\Xi_{k}$ satisfies $k^{*}=k$ and thus $\boldsymbol{b}=\boldsymbol{e}$.

The above lemma says that the $k$-th equality of (7) includes $k$ variables $\lambda_{k}, \lambda_{k-1}, \ldots, \lambda_{1}$ and is a linear equality with respect to $\lambda_{k}$. Thus, the equality system (7) has a unique solution.

Theorem 3.2. Equality system (7) has a unique solution $\left(\lambda_{1}, \lambda_{2}, \ldots, \lambda_{m}\right)$ satisfying $\lambda_{k}>0$ for every $k \in\{1,2, \ldots, m\}$.

A proof is given in appendix section.

Given a pair of Bernoulli sequences $\mathcal{X}$ and $\mathcal{X}^{\prime}$, we denote $\mathcal{X} \cdot \succ \mathcal{X}^{\prime}$, if and only if $\exists i \in\{1,2, \ldots, N\}$, satisfying $\mathcal{X}=\left(X_{1}, X_{2}, \ldots, X_{i}, \ldots, X_{N}\right), \mathcal{X}^{\prime}=$ 
$\left(X_{1}, X_{2}, \ldots, X_{i-1}, X_{i}^{\prime}, X_{i}^{\prime \prime}, X_{i+1}, \ldots, X_{N}\right)$, and $\mathbb{P}\left[X_{i}=0\right]=\mathbb{P}\left[X_{i}^{\prime}=0\right] \mathbb{P}\left[X_{i}^{\prime \prime}=\right.$ $0]$. We say that a Bernoulli sequences $\mathcal{X}^{\prime \prime}$ is a subdivision of $\mathcal{X}$, denoted by $\mathcal{X} \succeq \mathcal{X}^{\prime \prime}$, if and only if either $\mathcal{X}$ is equivalent to $\mathcal{X}^{\prime \prime}$ or there exists a finite sequence $\mathcal{X} \cdot \succ \mathcal{X}^{1} \succ \succ \mathcal{X}^{2} \cdot \succ \cdots \succ \mathcal{X}^{\prime \prime}$.

Now we consider the $m$-stopping odds problems defined on $\mathcal{X}$ and $\mathcal{X}^{\prime}$ satisfying $\mathcal{X} \succeq \mathcal{X}^{\prime}$

Lemma 3.3. Let $\mathcal{X}$ be a Bernoulli sequence and $\mathcal{X}^{\prime}$ be a subdivision of $\mathcal{X}$, i.e., $\mathcal{X} \succeq \mathcal{X}^{\prime}$. Odds problems with m-stoppings defined on $\mathcal{X}$ and $\mathcal{X}^{\prime}$ satisfies that the probability of win of an optimal strategy for $\mathcal{X}$ is greater than or equal to that of $\mathcal{X}^{\prime}$.

Proof. Obviously, we only need to consider the case that $\mathcal{X} \cdot \succ \mathcal{X}^{\prime}$. In the following, we show that there exists a strategy for $\mathcal{X}$ whose probability of win is equivalent to that of a (fixed) optimal strategy for $\mathcal{X}^{\prime}$.

Since $\mathcal{X} \cdot \succ \mathcal{X}^{\prime}$, there exists an index $i \in\{1,2, \ldots, N\}$, satisfying $\mathcal{X}=$ $\left(X_{1}, X_{2}, \ldots X_{N}\right), \mathcal{X}^{\prime}=\left(X_{1}, X_{2}, \ldots, X_{i-1}, X_{i}^{\prime}, X_{i}^{\prime \prime}, X_{i+1}, \ldots, X_{N}\right)$, and $q=q^{\prime} q^{\prime \prime}$, where $q=\mathbb{P}\left[X_{i}=0\right], q^{\prime}=\mathbb{P}\left[X_{i}^{\prime}=0\right], q^{\prime \prime}=\mathbb{P}\left[X_{i}^{\prime \prime}=0\right]$.

When we observe variables $\left(X_{1}, X_{2}, \ldots, X_{i-1}\right)$ in $\mathcal{X}$, we apply the optimal strategy for $\mathcal{X}^{\prime}$ and halt just before observing $X_{i}$.

(1) If $X_{i}=0$, then we do not select $X_{i}$ and put $\left(X_{i}^{\prime}, X_{i}^{\prime \prime}\right)=(0,0)$. We re-start the optimal strategy for $\mathcal{X}^{\prime}$ and apply to observed sequence of random variables $\left(X_{i+1}, \ldots, X_{N}\right)$.

(2) In case that $X_{i}=1$, we choose $\left(X_{i}^{\prime}, X_{i}^{\prime \prime}\right) \in\{(1,0),(0,1),(1,1)\}$ according to the following conditional probabilities;

$$
\left(X_{i}^{\prime}, X_{i}^{\prime \prime}\right)=\left\{\begin{array}{rrr}
(1,0) & \text { with probability } & \left(1-q^{\prime}\right) q^{\prime \prime} /\left(1-q^{\prime} q^{\prime \prime}\right) \\
(0,1) & \text { with probability } & q^{\prime}\left(1-q^{\prime \prime}\right) /\left(1-q^{\prime} q^{\prime \prime}\right) \\
(1,1) & \text { with probability } & \left(1-q^{\prime}\right)\left(1-q^{\prime \prime}\right) /\left(1-q^{\prime} q^{\prime \prime}\right)
\end{array}\right.
$$

We apply the optimal strategy for $\mathcal{X}^{\prime}$ to the chosen vector of $\left(X_{i}^{\prime}, X_{i}^{\prime \prime}\right)$. If the optimal strategy for $\mathcal{X}^{\prime}$ selects the last success in $\left(X_{i}^{\prime}, X_{i}^{\prime \prime}\right)$, we also select $X_{i}$ in $\mathcal{X}$ and vise verse. We re-start the optimal strategy for $\mathcal{X}^{\prime}$ and apply to observed sequence of random variables $\left(X_{i+1}, \ldots, X_{N}\right)$.

It is clear that, the strategy for $\mathcal{X}$, described above, selects the last success of $\mathcal{X}$, if and only if the optimal strategy for $\mathcal{X}^{\prime}$ selects the last success of $\mathcal{X}^{\prime}$. Thus, the probability of win of the above strategy is equivalent to that of the optimal strategy for $\mathcal{X}^{\prime}$.

Theorem 3.4. Let $\left(\lambda_{1}, \lambda_{2}, \ldots, \lambda_{m}\right)$ be a unique solution of equality system $(7)$. If a Bernoulli sequence $X_{1}, X_{2}, \ldots, X_{N}$ satisfies $\prod_{i=1}^{N} q_{i}<e^{-\sum_{k=1}^{m} \lambda_{k}}$, then the probability of win of an optimal strategy for the $m$-stopping odds problem defined on $X_{1}, X_{2}, \ldots, X_{N}$ is greater than or equal to

$$
\sum_{k=1}^{m} e^{-\sum_{k^{\prime}=1}^{k} \lambda_{k^{\prime}}}=e^{-\lambda_{1}}+e^{-\left(\lambda_{1}+\lambda_{2}\right)}+e^{-\left(\lambda_{1}+\lambda_{2}+\lambda_{3}\right)}+\cdots+e^{-\left(\lambda_{1}+\cdots+\lambda_{m}\right)} .
$$


This theorem says that; when $m=3,4,5$, our lower bounds are $e^{-1}+$ $e^{-\frac{3}{2}}+e^{-\frac{47}{24}} \geq 0.7321029820, e^{-1}+e^{-\frac{3}{2}}+e^{-\frac{47}{24}}+e^{-\frac{2761}{1152}} \geq 0.8231206726$, and $e^{-1}+e^{-\frac{3}{2}}+e^{-\frac{47}{24}}+e^{-\frac{2761}{1152}}+e^{-\frac{4162637}{1474560}} \geq 0.8825499145$, respectively. Table 1 shows our lower bounds in cases $m \leq 10$.

Proof. Let $\mathcal{X}^{0}=\left(X_{1}, X_{2}, \ldots, X_{N}\right)$ be a given Bernoulli sequence. We construct a sequences $\mathcal{X}^{1}$ satisfying $\mathcal{X}^{0} \cdot \succ \mathcal{X}^{1}$ by splitting a variable $X_{i}$ in $\mathcal{X}^{0}$ which attains the minimum $\min \left\{q_{1}, q_{2}, \ldots, q_{N}\right\}$, and introducing two random variables $X_{i}^{\prime}, X_{i}^{\prime \prime}$ satisfying $\mathbb{P}\left[X_{i}^{\prime}=0\right]=\mathbb{P}\left[X_{i}^{\prime \prime}=0\right]=\sqrt{q_{i}}$. Applying the above procedure iteratively, we obtain an infinite sequence $\mathcal{X}^{0} \cdot \succ \mathcal{X}^{1} \cdot \succ \mathcal{X}^{2} \cdot \succ \cdots$. It is clear that the maximum of odds of variables in $\mathcal{X}^{d}$ approaches to 0 as $d \rightarrow \infty$.

Let $\left(\mathcal{Y}^{1}, \mathcal{Y}^{2}, \ldots\right)$ be a subsequence of $\left(\mathcal{X}^{1}, \mathcal{X}^{2}, \ldots\right)$ satisfying that the maximum odds of $\mathcal{Y}^{d}$ is less than or equal to $2^{-d}$. We denote the Bernoulli sequence $\mathcal{Y}^{d}$ by $\left(Y_{1}^{d}, Y_{2}^{d}, \ldots, Y_{L_{d}}^{d}\right)$ and the corresponding odds by $\left(r_{1}^{(d)}, r_{2}^{(d)}, \ldots, r_{L_{d}}^{(d)}\right)$, where $L_{d}$ denotes the length of $\mathcal{Y}^{d}$. We introduce a specified threshold strategy $\operatorname{Threshold}\left(j_{d}^{(m)}, j_{d}^{(m-1)}, \ldots, j_{d}^{(1)}\right)$ for the $m$-stopping odds problem on $\left(Y_{1}^{d}, Y_{2}^{d}, \ldots, Y_{L_{d}}^{d}\right)$ defined by

$$
j_{d}^{(k)}=\min \left\{j \in\left\{1,2, \ldots, L_{d}\right\} \mid \lambda_{1}+\lambda_{2}+\cdots+\lambda_{k}>\sum_{j^{\prime}=j}^{L_{d}} r_{j^{\prime}}^{(d)}\right\},
$$

for each $k \in\{1,2, \ldots, m\}$. Theorem 3.2 implies inequalities $1 \leq j_{d}^{(m)} \leq$ $j_{d}^{(m-1)} \leq \cdots \leq j_{d}^{(1)} \leq L_{d}$ and thus we can define a corresponding threshold strategy. Let $\left\{B_{m+1}(d), B_{m}(d), \ldots, B_{1}(d)\right\}$ be a partition of index set $\left\{1,2, \ldots, L_{d}\right\}$ defined by

$$
B_{k}(d)= \begin{cases}\left\{j \in\left\{1,2, \ldots, L_{d}\right\} \mid j_{d}^{(1)} \leq j \leq L_{d}\right\} & (k=1), \\ \left\{j \in\left\{1,2, \ldots, L_{d}\right\} \mid j_{d}^{(k)} \leq j<j_{d}^{(k-1)}\right\} & (1<k \leq m), \\ \left\{j \in\left\{1,2, \ldots, L_{d}\right\} \mid 1 \leq j<j_{d}^{(m)}\right\} & (k=m+1) .\end{cases}
$$

Here we show that $1<j_{(d)}^{m}$. Since $\mathcal{Y}^{d}$ is a subdivision of $\mathcal{X}$, the probabilities of failure $q_{i}^{(d)}=\mathbb{P}\left[Y_{i}^{d}=0\right]$ and $q_{i}=\mathbb{P}\left[X_{i}=0\right]$ satisfy $q_{1}^{(d)} q_{2}^{(d)} \cdots q_{L_{d}}^{(d)}=$ $q_{1} q_{2} \cdots q_{N}$. The total sum of odds of $\mathcal{Y}^{d}$ satisfies that

$$
\begin{aligned}
\sum_{i=1}^{L_{d}} r_{i}^{(d)} & =\sum_{i=1}^{L_{d}}\left(\frac{1}{q_{i}^{(d)}}-1\right)=\sum_{i=1}^{L_{d}}\left(\frac{1}{q_{i}^{(d)}}\right)-L_{d} \geq L_{d}\left(\prod_{i=1}^{L_{d}} \frac{1}{q_{i}^{(d)}}\right)^{\frac{1}{L_{d}}}-L_{d}=L_{d}\left(\frac{1}{\prod_{i=1}^{L_{d}} q_{i}^{(d)}}\right)^{\frac{1}{L_{d}}}-L_{d} \\
& =L_{d}\left(\frac{1}{\prod_{i=1}^{N} q_{i}}\right)^{\frac{1}{L_{d}}}-L_{d}>L_{d}\left(\frac{1}{e^{-\sum_{k=1}^{m} \lambda_{k}}}\right)^{\frac{1}{L_{d}}}-L_{d}=L_{d}\left(\left(e^{\sum_{k=1}^{m} \lambda_{k}}\right)^{\frac{1}{L_{d}}}-1\right) \\
& \geq \lim _{L^{\prime} \rightarrow \infty} L^{\prime}\left(\left(e^{\sum_{k=1}^{m} \lambda_{k}}\right)^{\frac{1}{L^{\prime}}}-1\right)=\ln \left(e^{\sum_{k=1}^{m} \lambda_{k}}\right)=\sum_{k=1}^{m} \lambda_{k} .
\end{aligned}
$$

From the above, the total sum of odds of $\mathcal{Y}^{d}$ is strictly greater than $\sum_{k=1}^{m} \lambda_{k}$ and thus $1<j_{(d)}^{m}$, which implies $1 \in B_{m+1}(d) \neq \emptyset$. 
(i) First, we show that

$$
\lim _{d \rightarrow \infty} \sum_{i \in B_{k}(d)} r_{i}^{(d)}=\lambda_{k} \text { and } \lim _{d \rightarrow \infty}\left|B_{k}(d)\right|=+\infty, \quad \forall k \in\{1,2, \ldots, m\} .
$$

Since the odds of $\mathcal{Y}^{d}$ is less than $2^{-d}$, block $B_{k}(d)$ satisfies

$$
\lambda_{k}-2^{-d} \leq \sum_{i \in B_{k}(d)} r_{i}^{(d)} \leq \lambda_{k}+2^{-d}(\forall k \in\{1,2, \ldots, m\})
$$

and thus, we obtain the first equality in (9). Since $\lambda_{k}-2^{-d} \leq \sum_{i \in B_{k}(d)} r_{i}^{(d)} \leq$ $\left|B_{k}(d)\right| 2^{-d}$, it is clear that $\lim _{d \rightarrow \infty}\left|B_{k}(d)\right| \geq \lim _{d \rightarrow \infty}\left(2^{d} \lambda_{k}-1\right)=+\infty$.

(ii) It is easy to show that for any $k \in\{1,2, \ldots, m\}$,

$$
\begin{aligned}
\lim _{d \rightarrow \infty} \prod_{j \in B_{k}(d)} q_{i}^{(d)} & =\lim _{d \rightarrow \infty} \prod_{j \in B_{k}(d)}\left(\frac{1}{1+r_{i}^{(d)}}\right)=\lim _{d \rightarrow \infty}\left(\prod_{j \in B_{k}(d)}\left(1+r_{i}^{(d)}\right)\right)^{-1} \\
& \geq \lim _{d \rightarrow \infty}\left(\frac{\sum_{j \in B_{k}(d)}\left(1+r_{i}^{(d)}\right)}{\left|B_{k}(d)\right|}\right)^{-\left|B_{k}(d)\right|}=\lim _{d \rightarrow \infty}\left(1+\frac{\sum_{j \in B_{k}(d)} r_{i}^{(d)}}{\left|B_{k}(d)\right|}\right)^{-\left|B_{k}(d)\right|}=e^{-\lambda_{k}}
\end{aligned}
$$

(iii) Third, we show that the elementary symmetric polynomials satisfy

$$
\forall k \in\{1,2, \ldots, m\}, \forall b \in\{0,1, \ldots, m\}, \lim _{d \rightarrow \infty} f^{b}\left(B_{k}(d)\right) \geq \frac{\lambda_{k}^{b}}{b !} .
$$

When $b=0$, the definition of $f^{0}\left(B_{k}(d)\right)$ says that $f^{0}\left(B_{k}(d)\right)=1=\frac{\lambda_{k}^{0}}{0 !}$ holds permanently. Let us consider the cases that $b \geq 1$. When $d$ is sufficiently large, the size of $B_{k}(d)$ is greater than $m$, since $\lim _{d \rightarrow \infty}\left|B_{k}(d)\right|=+\infty$. Thus, we only need to consider the case that $f^{b}\left(B_{k}(d)\right)=\sum_{B^{\prime} \subseteq B_{k}(d),\left|B^{\prime}\right|=b}\left(\prod_{i \in B^{\prime}} r_{i}^{(d)}\right)$. If we introduce a vector of variables $\boldsymbol{r}^{\prime}=\left(r_{1}^{\prime}, r_{2}^{\prime}, \ldots, r_{j}^{\prime}\right)$ where $j=\left|B_{k}(d)\right|$, then the value $f^{b}\left(B_{k}(d)\right)$ is bounded from below by the optimal value of an optimization problem

$\min \left\{f^{b}\left(\boldsymbol{r}^{\prime}\right) \mid 0 \leq r_{i}^{\prime} \leq 2^{-d}(\forall i \in\{1,2, \ldots, j\}), \quad r_{1}^{\prime}+r_{2}^{\prime}+\cdots+r_{j}^{\prime} \geq \lambda_{k}-2^{-d}\right\}$,

where $f^{b}\left(\boldsymbol{r}^{\prime}\right)=\sum_{1 \leq i_{1}<i_{2}<\cdots<i_{b} \leq j} r_{i_{1}}^{\prime} r_{i_{2}}^{\prime} \cdots r_{i_{b}}^{\prime}$ and the last inequality constraint is obtained from (10). The optimization problem (11) minimizes a continuous function over a bounded closed set (a compact set) and has an optimal solution, since a given vector of odds $\left(r_{i}^{(d)} \mid i \in B_{k}(d)\right)$ (arranged in increasing order of indices) is feasible for (11). Let $\boldsymbol{r}^{*}=\left(r_{1}^{*}, r_{2}^{*}, \ldots, r_{j}^{*}\right)$ be an optimal solution of (11). From the symmetry of the objective function and the feasible region, we can assume that $\boldsymbol{r}^{*}$ satisfies $r_{1}^{*} \geq r_{2}^{*} \geq \cdots \geq r_{j}^{*}$. Now we show that if $r_{i+1}^{*}>0$, then $r_{i}^{*}=2^{-d}$. Assume on the contrary that $2^{-d}>r_{i}^{*}$ and $r_{i+1}^{*}>0$ hold. Then 
the vector $\left(r_{1}^{*}, r_{2}^{*}, \ldots, r_{i}^{*}+\varepsilon, r_{i+1}^{*}-\varepsilon, r_{i+2}^{*}, \ldots, r_{j}^{*}\right)$ is feasible to (11) and strictly decreases the objective function value, for some sufficiently small positive $\varepsilon$. This contradicts with the optimality of $\boldsymbol{r}^{*}$. Thus, the optimal solution $\boldsymbol{r}^{*}$ is denoted by $\left(2^{-d}, 2^{-d}, \ldots, 2^{-d}, r^{\prime \prime}, 0, \ldots, 0\right)$ where $0 \leq r^{\prime \prime} \leq 2^{-d}$. It is easy to see that $\boldsymbol{r}^{*}$ satisfies the last constraint with equality, i.e., $r_{1}^{*}+r_{2}^{*}+\cdots+r_{j}^{*}=\lambda_{k}-2^{-d}$. Consequently, the number of non-zero elements in $r^{*}$ is greater than or equal to $2^{d} \lambda_{k}-1$. Thus, we have that $\forall b \in\{1,2, \ldots, m\}$,

$$
\begin{aligned}
\lim _{d \rightarrow \infty} f^{b}\left(B_{k}(d)\right) & \geq \lim _{d \rightarrow \infty} f^{b}\left(\boldsymbol{r}^{*}\right) \geq \lim _{d \rightarrow \infty}\left(\begin{array}{c}
2^{d} \lambda_{k}-2 \\
b
\end{array}\right)\left(2^{-d}\right)^{b} \\
& \geq \lim _{d \rightarrow \infty} \frac{\left(2^{d} \lambda_{k}-2-b\right)^{b}}{b !}\left(2^{-d}\right)^{b}=\lim _{d \rightarrow \infty} \frac{\left(\lambda_{k}-(2+b) / 2^{d}\right)^{b}}{b !}=\frac{\lambda_{k}^{b}}{b !} .
\end{aligned}
$$

(iv) Lastly, we show our lower bound. As shown in Lemma 3.3, for any positive integer $d$, the probability of win of any threshold strategy for the $m$-stopping odds problem defined on $\mathcal{Y}^{d}$ gives a lower bound of that defined on the given sequence $\mathcal{X}$. Lower bounds derived in (ii) and (iii) directly imply that the win probability of Threshold $\left(j_{d}^{(m)}, j_{d}^{(m-1)}, \ldots, j_{d}^{(1)}\right)$ gives a lower bound

$$
\begin{aligned}
& \lim _{d \rightarrow \infty} \sum_{k=1}^{m}\left(\left(\prod_{i \in B_{k} \cup \cdots \cup B_{2} \cup B_{1}} q_{i}\right) \sum_{\left(b_{k}, \ldots, b_{1}\right) \in \Xi_{k}}\left(f^{b_{k}}\left(B_{k}(d)\right) \cdots f^{b_{1}}\left(B_{1}(d)\right)\right)\right) \\
& \geq \sum_{k=1}^{m}\left(\left(\prod_{k^{\prime}=1}^{k} e^{-\lambda_{k^{\prime}}}\right)\left(\sum_{\left(b_{k}, \ldots, b_{1}\right) \in \Xi_{k}} \frac{\lambda_{k}^{b_{k}}}{b_{k} !} \frac{\lambda_{k-1}^{b_{k-1}}}{b_{k-1} !} \cdots \frac{\lambda_{1}^{b_{1}}}{b_{1} !}\right)\right)=\sum_{k=1}^{m} e^{-\sum_{k^{\prime}=1}^{k} \lambda_{k^{\prime}}}
\end{aligned}
$$

where the last equality is obtained from (7).

\section{Secretary Problem.}

In this section, we show that an optimal strategy for the secretary problem attains our lower bounds appearing in Theorem 3.4. We discuss a sequence of $0 / 1$ random variables $X_{2}, X_{3}, \ldots, X_{N}$ satisfying $\mathbb{P}\left[X_{i}=1\right]=1 / i$, for any $i \in\{2,3, \ldots, N\}$. In this section, $q_{i}$ denotes the probability of failure $1-1 / i$ and $r_{i}$ denotes the odds $1 /(i-1)$ of $X_{i}$, for all $i \in\{1,2, \ldots, N\}$. Gilbert and Mosteller Gilbert and Mosteller (1966) showed that an optimal strategy for the secretary problem with $m$-stoppings is attained by a threshold strategy.

First, we show some properties related to the optimal threshold strategy.

Lemma 4.1. Consider an m-stopping secretary problem defined on Bernoulli sequence $X_{2}, X_{3}, \ldots, X_{N}$ satisfying $\mathbb{P}\left[X_{i}=1\right]=1 / i(\forall i \in\{2,3, \ldots, N\})$. We denote an optimal threshold strategy by $\operatorname{Threshold}\left(i_{N}^{(m)}, i_{N}^{(m-1)}, \ldots, i_{N}^{(1)}\right)$, and define a block partition $\left\{B_{m+1}(N), B_{m}(N), \ldots, B_{1}(N)\right\}$ of index set $\{2,3, \ldots, N\}$ 
Table 1: Cumulative sum of entries in $\left(\lambda_{1}, \lambda_{2}, \ldots, \lambda_{m}\right)$ and lower bounds.

\begin{tabular}{|c|c|c|c|c|}
\hline$m$ & $\sum_{k=1}^{m} \lambda_{k}$ & & & $\sum_{k=1}^{m} e^{-\sum_{k^{\prime}=1}^{k} \lambda_{k^{\prime}}}$ \\
\hline 1 & $\begin{array}{ll}k=1 \\
1\end{array}$ & $=$ & 1 & $0.3678794411 \cdots$ \\
\hline 2 & $3 / 2$ & $=$ & 1.5 & $0.5910096013 \ldots$ \\
\hline 3 & $47 / 24$ & $=$ & $1.958333 \cdots$ & $0.7321029820 \cdots$ \\
\hline 4 & $2761 / 1152$ & $=$ & $2.396701 \cdots$ & $0.8231206726 \cdots$ \\
\hline 5 & $4162637 / 1474560$ & $=$ & $2.822969 \ldots$ & $0.8825499145 \cdots$ \\
\hline 6 & $380537052235603 / 117413668454400$ & $=$ & $3.240994 \cdots$ & $0.9216748810 \cdots$ \\
\hline 7 & $\frac{705040594914523588948186792543}{193003573558876719588311040000}$ & \multicolumn{2}{|c|}{$=3.652992 \cdots$} & $0.9475883491 \cdots$ \\
\hline \multirow{2}{*}{8} & $\begin{array}{r}3025002101 \\
77484374840641189918370275991590974715547528765249 \\
\end{array}$ & \multirow{2}{*}{\multicolumn{2}{|c|}{$=4.060364 \cdots$}} & \multirow{2}{*}{$0.9648310882 \cdots$} \\
\hline & $\begin{array}{rr}12993473612938854416966977838930799571763200000000 \\
\end{array}$ & & & \\
\hline \multirow{2}{*}{9} & $\begin{array}{r}4955429267826902943 \\
22991702889058732983678465397265103848504031927299 \\
12522937262239403638817695466470734534217406992001 \\
\end{array}$ & \multirow{2}{*}{\multicolumn{2}{|c|}{$=4.464059 \cdots$}} & \multirow{2}{*}{$0.9763466188 \cdots$} \\
\hline & $\begin{array}{rr}1110072612742364945 \\
47845493213273623476317581768829551455545915219181 \\
23315624957195621435513013513748480000000000000000\end{array}$ & & & \\
\hline \multirow{2}{*}{10} & $\begin{array}{r}11989289035379212035246168789525873032 \\
80849078486814692748999764352069320540924554366342 \\
20167531781129657310860185112917637526070528528590 \\
30333616681207477435841890935057636581590554638168 \\
66245450807944253110095088765765115912740477984001\end{array}$ & & \multirow{2}{*}{$4.864751 \cdots$} & \multirow{2}{*}{$0.9840603638 \cdots$} \\
\hline & $\begin{array}{r}2464522411121321065440235649497935699 \\
29104324006809890312129945082092425080632748539150 \\
55027199035483748007701106129537805807977992169375 \\
27132513498662936774805285136477456358447232852772 \\
52323755961404620800000000000000000000000000000000\end{array}$ & & & \\
\hline
\end{tabular}


by

$$
B_{k}(N)= \begin{cases}\left\{i \in\{2,3, \ldots, N\} \mid i_{N}^{(1)} \leq i \leq N\right\} & (k=1), \\ \left\{i \in\{2,3, \ldots, N\} \mid i_{N}^{(k)} \leq i<i_{N}^{(k-1)}\right\} & (1<k \leq m), \\ \left\{i \in\{2,3, \ldots, N\} \mid 2 \leq i<i_{N}^{(m)}\right\} & (k=m+1) .\end{cases}
$$

Then, for each $k \in\{1,2, \ldots, m\}$, the following properties hold; (i) $\lim _{N \rightarrow \infty} i_{N}^{(k)}=+\infty$, (ii) $\lim _{N \rightarrow \infty} \sum_{i \in B_{k}(N)} r_{i}=\lambda_{k}$, (iii) $\lim _{N \rightarrow \infty} \prod_{i \in B_{k}(N)} q_{i}=e^{-\lambda_{k}}$, and (iv) $\lim _{N \rightarrow \infty} f^{b}\left(B_{k}(N)\right)=$ $\frac{\lambda_{k}^{b}}{b !}(\forall b \in\{0,1, \ldots, m\})$ where $\left(\lambda_{1}, \lambda_{2}, \ldots, \lambda_{m}\right)$ is a unique solution of $(7)$.

Proof. It is well-known that for any $k \in\{1,2, \ldots, m\}$, the threshold strategy Threshold $\left(i_{N}^{(k)}, i_{N}^{(k-1)}, \ldots, i_{N}^{(1)}\right)$ is also optimal to the $k$-stopping secretary problem Gilbert and Mosteller (1966).

In the following, we show desired properties by induction on $k$. When $k=1$, the problem becomes the classical secretary problem, and properties (i), ..,(iv) are well-known (see Gilbert and Mosteller (1966) for example).

Now, we begin a discussion of the $k$-th induction step (where $k \leq m$ ) under an assumption that for any $k^{\prime} \in\{1,2, \ldots, k-1\}$, properties (i), ..,(iv) hold. Let $\boldsymbol{e}$ be a unit $k$-vector $(1,0,0, \ldots, 0)$. Lemma 3.1 says that $\boldsymbol{e} \in \Xi_{k}$ and every vector $\left(b_{k}, b_{k-1}, \ldots, b_{1}\right) \in \Xi_{k} \backslash\{\boldsymbol{e}\}$ satisfies $b_{k}=0$. Thus, the induction hypothesis (iv) and the definition of equality system (7) imply that

$$
\begin{aligned}
& \lim _{N \rightarrow \infty}\left(\sum_{\left(b_{k}, \ldots, b_{1}\right) \in \Xi_{k} \backslash\{\boldsymbol{e}\}}\left(f^{b_{k-1}}\left(B_{k-1}(N)\right) f^{b_{k-2}}\left(B_{k-2}(N)\right) \cdots f^{b_{1}}\left(B_{1}(N)\right)\right)\right) \\
& =\sum_{\left(b_{k}, \ldots, b_{1}\right) \in \Xi_{k} \backslash\{\boldsymbol{e}\}}\left(\frac{\lambda_{k-1}^{b_{k-1}}}{b_{k-1} !} \frac{\lambda_{k-2}^{b_{k-2}}}{b_{k-2} !} \cdots \frac{\lambda_{1}^{b_{1}}}{b_{1} !}\right)=\sum_{\left(b_{k}, \ldots, b_{1}\right) \in \Xi_{k} \backslash\{\boldsymbol{e}\}}\left(\frac{\lambda_{k}^{0}}{0 !} \frac{\lambda_{k-1}^{b_{k-1}}}{b_{k-1} !} \frac{\lambda_{k-2}^{b_{k-2}}}{b_{k-2} !} \cdots \frac{\lambda_{1}^{b_{1}}}{b_{1} !}\right)=1-\left(\lambda_{k}\right)
\end{aligned}
$$

Now we introduce a threshold strategy $\operatorname{Threshold}\left(i, i_{N}^{(k-1)}, i_{N}^{(k-2)}, \ldots, i_{N}^{(1)}\right)$ $\left(\forall i \in\left\{2,3, \ldots, i_{N}^{(k-1)}\right\}\right)$ for the $k$-stopping secretary problem and employ a onestage look-ahead approach Ano $(2001)$. Let $\mathbb{P}_{N}^{(\text {win })}(k, i)$ be a probability of win of the threshold strategy Threshold $\left(i, i_{N}^{(k-1)}, i_{N}^{(k-2)}, \ldots, i_{N}^{(1)}\right)$. 
A difference of a pair $\mathbb{P}_{N}^{(\text {win })}(k, i-1)$ and $\mathbb{P}_{N}^{(\text {win })}(k, i)$ satisfies

$$
\begin{aligned}
& \mathbb{P}_{N}^{(\text {win })}(k, i-1)-\mathbb{P}_{N}^{(\text {win })}(k, i) \\
& =\left(\prod_{i^{\prime}=i-1}^{N} q_{i^{\prime}}\right)\left(\sum_{i^{\prime}=i-1}^{i_{N}^{(k-1)}-1} r_{i^{\prime}}+\sum_{\left(b_{k}, \ldots, b_{1}\right) \in \Xi_{k} \backslash\{\boldsymbol{e}\}}\left(f^{b_{k-1}}\left(B_{k-1}(N)\right) \cdots f^{b_{1}}\left(B_{1}(N)\right)\right)\right) \\
& -\left(\prod_{i^{\prime}=i}^{N} q_{i^{\prime}}\right)\left(\sum_{i^{\prime}=i}^{i_{N}^{(k-1)}-1} r_{i^{\prime}}+\sum_{\left(b_{k}, \ldots, b_{1}\right) \in \Xi_{k} \backslash\{\boldsymbol{e}\}}\left(f^{b_{k-1}}\left(B_{k-1}(N)\right) \cdots f^{b_{1}}\left(B_{1}(N)\right)\right)\right)
\end{aligned}
$$

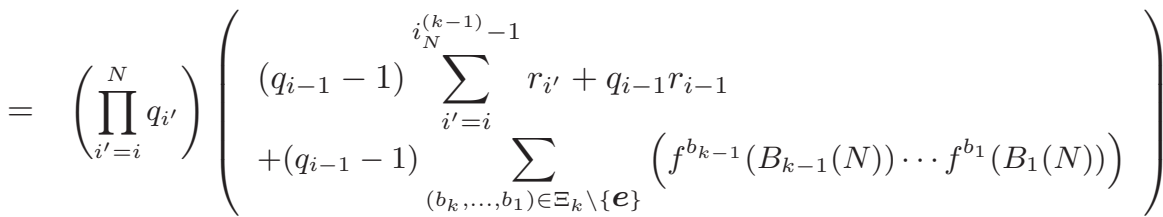

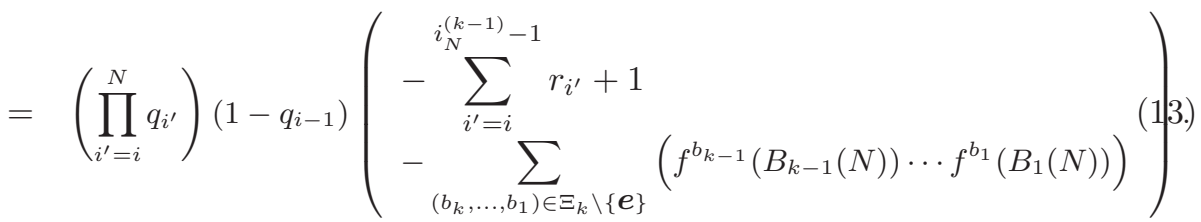

It is easy to show that

$\lim _{N \rightarrow \infty}\left(\mathbb{P}_{N}^{(\text {win })}(k, 3)-\mathbb{P}_{N}^{(\text {win })}(k, 2)\right)>0>\lim _{N \rightarrow \infty}\left(\mathbb{P}_{N}^{(\text {win })}\left(k, i_{N}^{(k-1)}\right)-\mathbb{P}_{N}^{(\text {win })}\left(k, i_{N}^{(k-1)}-1\right)\right)$, which implies that $\exists N^{\prime}, \forall N>N^{\prime}, 3 \leq i_{N}^{(k)} \leq i_{N}^{(k-1)}-1$. From the optimality of the threshold value $i_{N}^{(k)}$, inequalities $\mathbb{P}_{N}^{(\text {win })}\left(k, i_{N}^{(k)}-1\right) \leq \mathbb{P}_{N}^{(\text {win })}\left(k, i_{N}^{(k)}\right) \geq$ $\mathbb{P}_{N}^{(\text {win })}\left(k, i_{N}^{(k)}+1\right)$ hold.

(i) Since $0 \leq \mathbb{P}_{N}^{(\operatorname{win})}\left(k, i_{N}^{(k)}\right)-\mathbb{P}_{N}^{(\text {win })}\left(k, i_{N}^{(k)}+1\right)$, equality (13) implies that

$$
\sum_{i^{\prime}=i_{N}^{(k)}+1}^{i_{N}^{(k-1)}-1} r_{i^{\prime}} \leq 1-\sum_{\left(b_{k}, \ldots, b_{1}\right) \in \Xi_{k} \backslash\{\boldsymbol{e}\}}\left(f^{b_{k-1}}\left(B_{k-1}(N)\right) \cdots f^{b_{1}}\left(B_{1}(N)\right)\right) .
$$

As a consequence of the above inequality and (12), we have that

$$
\begin{aligned}
\lambda_{k} & =\lim _{N \rightarrow \infty}\left(1-\sum_{\left(b_{k}, \ldots, b_{1}\right) \in \Xi_{k} \backslash\{\boldsymbol{e}\}}\left(f^{b_{k-1}}\left(B_{k-1}(N)\right) \cdots f^{b_{1}}\left(B_{1}(N)\right)\right)\right) \geq \lim _{N \rightarrow \infty}\left(\sum_{i^{\prime}=i_{N}^{(k)}+1}^{i_{N}^{(k-1)}-1} r\left(i_{1} 14\right)\right. \\
& =\lim _{N \rightarrow \infty}\left(\sum_{i^{\prime}=i_{N}^{(k)}}^{i_{N}^{(k-1)}-2} \frac{1}{i^{\prime}}\right) \geq \lim _{N \rightarrow \infty}\left(\ln \frac{i_{N}^{(k-1)}-1}{i_{N}^{(k)}}\right) .
\end{aligned}
$$

The above inequality and the assumption that $\lim _{N \rightarrow \infty} i_{N}^{(k-1)}=+\infty$ imply the property that $\lim _{N \rightarrow \infty} i_{N}^{(k)}=+\infty$. 
(ii) Form inequality (14) and the property $\lim _{N \rightarrow \infty} i_{N}^{(k)}=+\infty$, we obtain that $\lim _{N \rightarrow \infty} \sum_{i^{\prime} \in B_{k}(N)} r_{i^{\prime}}=\lim _{N \rightarrow \infty} \sum_{i^{\prime}=i_{N}^{(k)}}^{i_{N}^{(k-1)}-1} r_{i^{\prime}}=\lim _{N \rightarrow \infty}\left(r_{i_{N}^{(k)}}+\sum_{i^{\prime}=i_{N}^{(k)}+1}^{i_{N}^{(k-1)}-1} r_{i^{\prime}}\right) \leq \lim _{N \rightarrow+\infty}\left(\frac{1}{i_{N}^{(k)}-1}\right)+\lambda_{k}=\lambda_{k}$.

The inequality $0 \geq \mathbb{P}_{N}^{(\text {win })}\left(k, i_{N}^{(k)}-1\right)-\mathbb{P}_{N}^{(\text {win })}\left(k, i_{N}^{(k)}\right)$ and equality (13) directly imply that

$$
\sum_{i^{\prime}=i_{N}^{(k)}}^{i_{N}^{(k-1)}-1} r_{i^{\prime}} \geq 1-\sum_{\left(b_{k}, \ldots, b_{1}\right) \in \Xi_{k} \backslash\{\boldsymbol{e}\}}\left(f^{b_{k-1}}\left(B_{k-1}(N)\right) \cdots f^{b_{1}}\left(B_{1}(N)\right)\right) .
$$

Combining the above inequality and (12), we obtain that

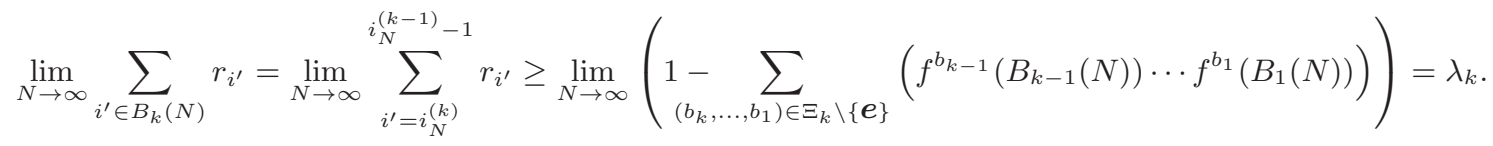

As a result, we have shown that $\lim _{N \rightarrow \infty} \sum_{i \in B_{k}(N)} r_{i}=\lambda_{k}$.

(iii) From the definition of $q_{i}$, it is clear that

$$
\begin{array}{r}
\ln \prod_{i \in B_{k}(N)} q_{i}=\sum_{i \in B_{k}(N)} \ln (1-1 / i)=\sum_{i \in B_{k}(N)}(\ln (i-1)-\ln i) \\
=\ln \left(i_{N}^{(k)}-1\right)-\ln \left(i_{N}^{(k-1)}-1\right)=\ln \frac{i_{N}^{(k)}-1}{i_{N}^{(k-1)}-1}
\end{array}
$$

and

$$
\sum_{i=i_{N}^{(k)}-1}^{i_{N}^{(k-1)}-2} \frac{1}{i} \geq \ln \left(i_{N}^{(k-1)}-1\right)-\ln \left(i_{N}^{(k)}-1\right) \geq \sum_{i=i_{N}^{(k)}}^{i_{N}^{(k-1)}-2} \frac{1}{i},
$$

where $i_{N}^{(0)}$ denotes $N+1$. From the above, we obtain that

$$
\begin{aligned}
& -\lim _{N \rightarrow \infty} \ln \prod_{i \in B_{k}(N)} q_{i} \leq \lim _{N \rightarrow \infty} \sum_{i=i_{N}^{(k)}-1}^{i_{N}^{(k-1)}-2} \frac{1}{i}=\lim _{N \rightarrow \infty} \sum_{i=i_{N}^{(k)}}^{i_{N}^{(k-1)}-1} r_{i}=\lim _{N \rightarrow \infty} \sum_{i=B_{k}(N)} r_{i}=\lambda_{k}, \quad \text { and } \\
& -\lim _{N \rightarrow \infty} \ln \prod_{i \in B_{k}(N)} q_{i} \geq \lim _{N \rightarrow \infty} \sum_{i=i_{N}^{(k)}}^{i_{N}^{(k-1)}-2} \frac{1}{i}=\lim _{N \rightarrow \infty}\left(\left(\frac{-1}{i_{N}^{(k)}-1}\right)+\sum_{i=i_{N}^{(k)}}^{i_{N}^{(k-1)}-1} r_{i}\right)=\lim _{N \rightarrow \infty} \sum_{i=B_{k}(N)} r_{i}=\lambda_{k} .
\end{aligned}
$$

Accordingly, we have that $\lim _{N \rightarrow \infty} \prod_{i \in B_{k}(N)} q_{i}=e^{-\lambda_{k}}$. 
(iv) We omit the case of $f^{0}\left(B_{k}(N)\right)$ in the following, since the equality $f^{0}\left(B_{k}(N)\right)=$ $1=\frac{\lambda_{k}^{0}}{0 !}$ holds permanently. We discuss cases $b \in\{1,2, \ldots, m\}$. The size of block $B_{k}(N)$ satisfies the following;

$$
\lim _{N \rightarrow \infty}\left|B_{k}(N)\right| r_{i_{N}^{(k)}} \geq \lim _{N \rightarrow \infty} \sum_{i=i_{N}^{(k)}}^{i_{N}^{(k-1)}-1} r_{i}=\lim _{N \rightarrow \infty} \sum_{i \in B_{k}(N)} r_{i}=\lambda_{k}>0
$$

The positivity of $\lambda_{k}$ and the equality $\lim _{N \rightarrow \infty} r_{i_{N}^{(k)}}=\lim _{N \rightarrow \infty} \frac{1}{i_{N}^{(k)}-1}=0$ imply that $\lim _{N \rightarrow \infty}\left|B_{k}(N)\right|=+\infty$. Thus, we have that $\exists N^{\prime}, \forall N>N^{\prime}$, the size of $B_{k}(N)$ exceeds $m$ and thus

$$
\forall b \in\{1,2, \ldots, m\}, \quad f^{b}\left(B_{k}(N)\right)=\sum_{B^{\prime} \subseteq B_{k}(N),\left|B^{\prime}\right|=b}\left(\prod_{i \in B^{\prime}} r_{i}\right) .
$$

We show that $\lim _{N \rightarrow \infty} f^{b}\left(B_{k}(N)\right)=\frac{\lambda_{k}^{b}}{b !}$ by induction on $b$. When $b=1$, property (ii) implies that

$$
\lim _{N \rightarrow \infty} f^{1}\left(B_{k}(N)\right)=\lim _{N \rightarrow \infty} \sum_{i \in B_{k}(N)} r_{i}=\frac{\lambda_{k}^{1}}{1 !}
$$

For any positive vector $\boldsymbol{r}^{\prime} \in \mathbb{R}^{n^{\prime}}$, the inequality $f^{b}\left(\boldsymbol{r}^{\prime}\right) \leq\left(\sum_{i=1}^{n^{\prime}} r_{i}^{\prime}\right) /(b !)$ holds, and thus we have

$$
\lim _{N \rightarrow \infty} f^{b}\left(B_{k}(N)\right) \leq \lim _{N \rightarrow \infty} \frac{\left(\sum_{i \in B_{k}(N)} r_{i}\right)^{b}}{b !}=\frac{\lambda_{k}^{b}}{b !} .
$$

The induction hypothesis $\lim _{N \rightarrow \infty} f^{b-1}\left(B_{k}(N)\right)=\frac{\lambda_{k}^{b-1}}{(b-1) !}$ implies that

$$
\begin{aligned}
& \lim _{N \rightarrow \infty} f^{b}\left(B_{k}(N)\right)=\lim _{N \rightarrow \infty} \sum_{B^{\prime} \subseteq B_{k}(N),\left|B^{\prime}\right|=b}\left(\prod_{i \in B^{\prime}} r_{i}\right) \\
& \left.=\lim _{N \rightarrow \infty}\left(\frac{1}{b}\right) \sum_{B^{\prime \prime} \subseteq B_{k}(N),\left|B^{\prime \prime}\right|=b-1}\left(\prod_{i \in B^{\prime \prime}} r_{i}\right)\left(\sum_{j \in B_{k}(B) \backslash B^{\prime \prime}} r_{j}\right)\right) \\
& \geq \lim _{N \rightarrow \infty}\left(\frac{1}{b}\right)\left(\sum_{i \in B_{k}(N)} r_{i}-(b-1) r_{i_{N}^{(k)}} \sum_{B^{\prime \prime} \subseteq B_{k}(N),\left|B^{\prime \prime}\right|=b-1}\left(\prod_{i \in B^{\prime \prime}} r_{i}\right)\right. \\
& =\lim _{N \rightarrow \infty} \frac{\left(\sum_{i \in B_{k}(N)} r_{i}-(b-1) r_{i_{N}^{(k)}}\right)}{b} f^{b-1}\left(B_{k}(N)\right)=\frac{\lambda_{k}}{b} \frac{\lambda_{k}^{b-1}}{(b-1) !}=\frac{\left.\lambda_{k}^{b} 17\right)}{b !}
\end{aligned}
$$

Thus, we have shown $\lim _{N \rightarrow \infty} f^{b}\left(B_{k}(N)\right)=\frac{\lambda_{k}^{b}}{b !}$.

The following theorem gives the win probability of the secretary problem. 
Theorem 4.2. Let $\left(\lambda_{1}, \lambda_{2}, \ldots, \lambda_{m}\right)$ be a unique solution of (7). Given a sequence of $0 / 1$ random variables $X_{2}, X_{3}, \ldots, X_{N}$ satisfying $\mathbb{P}\left[X_{i}=1\right]=1 / i$ $(\forall i \in\{2,3, \ldots, N\})$, the probability of win $\mathbb{P}_{N}^{(\text {win) }}(m)$ of an optimal strategy for the $m$-stopping secretary problem defined on $X_{2}, X_{3}, \ldots, X_{N}$ satisfies

$$
\lim _{N \rightarrow \infty} \mathbb{P}_{N}^{(\text {win) }}(m)=\sum_{k=1}^{m} e^{-\sum_{k^{\prime}=1}^{k} \lambda_{k^{\prime}}}
$$

Proof. In the previous lemma, we have shown properties (iii) and (iv) for each $k \in\{1,2, \ldots, m\}$. Thus, the probability of win $\mathbb{P}_{N}^{(\text {win })}(m)$ satisfies

$$
\begin{aligned}
\lim _{N \rightarrow \infty} \mathbb{P}_{N}^{(\operatorname{win})}(m) & \left.=\lim _{N \rightarrow \infty} \sum_{k=1}^{m}\left(\prod_{i \in B_{k}(N) \cup \cdots \cup B_{1}(N)} q_{i}\right)\left(\sum_{\left(b_{k}, \ldots, b_{1}\right) \in \Xi_{k}}\left(f^{b_{k}}\left(B_{k}(N)\right) \cdots f^{b_{1}}\left(B_{1}(N)\right)\right)\right)\right) \\
& =\sum_{k=1}^{m}\left(\left(\prod_{k^{\prime}=1}^{k} e^{-\lambda_{k^{\prime}}}\right)\left(\sum_{\left(b_{k}, \ldots, b_{1}\right) \in \Xi_{k}} \frac{\lambda_{k}^{b_{k}}}{b_{k} !} \frac{\lambda_{k-1}^{b_{k-1}}}{b_{k-1} !} \cdots \frac{\lambda_{1}^{b_{1}}}{b_{1} !}\right)\right)=\sum_{k=1}^{m} e^{-\sum_{k^{\prime}=1}^{k} \lambda_{k^{\prime}}},
\end{aligned}
$$

where the last equality is obtained from (7).

In the rest of this section, we prove a conjecture on a relation between threshold values and win probability of the secretary problem indicated by Gilbert and Mosteller Gilbert and Mosteller (1966).

Theorem 4.3. Given a sequence of $0 / 1$ random variables $X_{2}, X_{3}, \ldots, X_{N}$ satisfying $\mathbb{P}\left[X_{i}=1\right]=1 / i(\forall i \in\{2,3, \ldots, N\})$, an optimal (threshold) strategy $\operatorname{Threshold}\left(i_{N}^{(m)}, i_{N}^{(m-1)}, \ldots, i_{N}^{(1)}\right)$ for the $m$-stopping secretary problem satisfies

$$
\lim _{N \rightarrow \infty}\left(\frac{i_{N}^{(m)}}{N}+\frac{i_{N}^{(m-1)}}{N}+\cdots+\frac{i_{N}^{(1)}}{N}\right)=\lim _{N \rightarrow \infty} \mathbb{P}_{N}^{(\text {win })}(m)
$$

where $\mathbb{P}_{N}^{(\text {win) }}(m)$ denotes a corresponding probability of win.

Proof. In the following, we put $i_{N}^{(0)}=N+1$ and we denotes a unique solution of (7) by $\left(\lambda_{1}, \lambda_{2}, \ldots, \lambda_{m}\right)$. Equality (15) imply that for any $k \in\{1,2, \ldots, m\}$,

$$
\begin{aligned}
& \lim _{N \rightarrow \infty} \ln \frac{i_{N}^{(k)}}{i_{N}^{(k-1)}}=\lim _{N \rightarrow \infty} \ln \left(\frac{i_{N}^{(k)}-1}{i_{N}^{(k-1)}-1} \frac{i_{N}^{(k-1)}-1}{i_{N}^{(k-1)}} \frac{i_{N}^{(k)}}{i_{N}^{(k)}-1}\right) \\
& =\lim _{N \rightarrow \infty}\left(\ln \left(\prod_{i \in B_{k}(N)} q_{i}\right)+\ln \left(1-\frac{1}{i_{N}^{(k-1)}}\right)+\ln \left(1+\frac{1}{i_{N}^{(k)}-1}\right)\right)=-\lambda_{k} .
\end{aligned}
$$


Thus, we have

$$
\begin{aligned}
\lim _{N \rightarrow \infty} \ln \frac{i_{N}^{(k)}}{N} & =\lim _{N \rightarrow \infty} \ln \left(\left(\frac{i_{N}^{(k)}}{i_{N}^{(k-1)}}\right)\left(\frac{i_{N}^{(k-1)}}{i_{N}^{(k-2)}}\right) \cdots\left(\frac{i_{N}^{(1)}}{i_{N}^{(0)}}\right)\left(\frac{N+1}{N}\right)\right) \\
& =\lim _{N \rightarrow \infty}\left(\ln \left(\frac{i_{N}^{(k)}}{i_{N}^{(k-1)}}\right)+\ln \left(\frac{i_{N}^{(k-1)}}{i_{N}^{(k-2)}}\right)+\cdots+\ln \left(\frac{i_{N}^{(1)}}{i_{N}^{(0)}}\right)+\ln \left(\frac{N+1}{N}\right)\right) \\
& =-\left(\lambda_{k}+\lambda_{k-1}+\cdots+\lambda_{1}\right)=-\sum_{k^{\prime}=1}^{k} \lambda_{k^{\prime}} .
\end{aligned}
$$

The above equality and Theorem 4.2 imply that

$$
\lim _{N \rightarrow \infty}\left(\frac{i_{N}^{(m)}}{N}+\frac{i_{N}^{(m-1)}}{N}+\cdots+\frac{i_{N}^{(1)}}{N}\right)=\lim _{N \rightarrow \infty} \sum_{k=1}^{m} \frac{i_{N}^{(k)}}{N}=\sum_{k=1}^{m} e^{-\sum_{k^{\prime}=1}^{k} \lambda_{k^{\prime}}}=\lim _{N \rightarrow \infty} \mathbb{P}_{N}^{(\operatorname{win})}(m) .
$$

\section{Discussion.}

We dealt with the odds problem and the secretary problem with multiple stopping chances. We derived a tight lower bound of the probability of win for odds problem and showed that the lower bound is attained by the secretary problem. We also proved a conjecture on the secretary problem which connects the optimal threshold strategy and the probability of win.

\section{APPENDIX}

Proof of Theorem 3.2

Before showing Theorem 3.2, we need some definitions and a lemma. For any pair of positive integers $\left(k, k^{\prime}\right)$ satisfying $m \geq k>k^{\prime} \geq 1$, we introduce

$$
\Xi_{k}^{0}\left(k^{\prime}\right)=\left\{\left(b_{k}, b_{k-1}, \ldots, b_{1}\right) \in \Xi_{k} \mid 0=b_{k}=b_{k-1}=\cdots=b_{k^{\prime}+1}\right\},
$$

where we define $\Xi_{k}^{0}(k)=\Xi_{k}$. We also denote

$$
\alpha\left(k, k^{\prime}\right)=\sum_{\left(b_{k}, \ldots, b_{1}\right) \in \Xi_{k}^{0}\left(k^{\prime}\right)}\left(\frac{\lambda_{k}^{b_{k}}}{b_{k} !} \frac{\lambda_{k-1}^{b_{k-1}}}{b_{k-1} !} \cdots \frac{\lambda_{1}^{b_{1}}}{b_{1} !}\right)=\sum_{\left(0, \ldots, 0, b_{k^{\prime}}, \ldots, b_{1}\right) \in \Xi_{k}^{0}\left(k^{\prime}\right)}\left(\frac{\lambda_{k^{\prime}}^{b_{k^{\prime}}}}{b_{k^{\prime}} !} \frac{\lambda_{k^{\prime}}^{b_{k^{\prime}}-1}}{b_{k^{\prime}-1} !} \cdots \frac{\lambda_{1}^{b_{1}}}{b_{1} !}\right),
$$

where $\left(\lambda_{m}, \lambda_{m-1}, \ldots, \lambda_{1}\right)$ is a solution of equality system $(7)$.

Lemma 5.1. For any integer $k^{\prime} \in\{1,2, \ldots, m\}, \alpha(\cdot, \cdot)$ satisfies the following inequalities

$$
1=\alpha\left(k^{\prime}, k^{\prime}\right)>\alpha\left(k^{\prime}+1, k^{\prime}\right)>\cdots>\alpha\left(m, k^{\prime}\right)>0 .
$$


Proof. When we consider $\alpha\left(k^{\prime}, k^{\prime}\right)$, equality $\Xi_{k^{\prime}}^{0}\left(k^{\prime}\right)=\Xi_{k^{\prime}}$ holds and thus (7) directly implies that

$$
\alpha\left(k^{\prime}, k^{\prime}\right)=\sum_{\left(b_{k^{\prime}}, \ldots, b_{1}\right) \in \Xi_{k^{\prime}}}\left(\frac{\lambda_{k^{\prime}}^{b_{k^{\prime}}}}{b_{k^{\prime}} !} \frac{\lambda_{k^{\prime}-1}^{b_{k^{\prime}}-1}}{b_{k^{\prime}-1} !} \cdots \frac{\lambda_{1}^{b_{1}}}{b_{1} !}\right)=1 .
$$

Next, we show the above inequalities by induction on $k^{\prime}$.

When $k^{\prime}=1$, it is clear that $\Xi_{k}^{0}(1)=\{(0,0, \cdots, 0, k)\}$ and thus $\alpha(k, 1)=$ $\frac{\lambda_{1}^{k}}{k !}=\frac{1}{k !}$ for each $k \in\{1,2, \ldots, m\}$, since $\lambda_{1}=1$. Thus, we have the inequality

$$
1=\alpha(1,1)>\alpha(2,1)>\cdots>\alpha(m, 1)>0 .
$$

Next, we consider a general case. Every vector $\left(0, \ldots, 0, b_{k^{\prime}}, \ldots, b_{1}\right) \in \Xi_{k}^{0}\left(k^{\prime}\right)$ satisfies that $b_{k^{\prime}} \in\left\{0,1, \ldots, 1+k-k^{\prime}\right\}$. Especially in the case that $b_{k^{\prime}}=$ $1+k-k^{\prime}$, the definition of $\Xi_{k}$ implies that $\left(0, \ldots, 0, b_{k^{\prime}}, \ldots, b_{1}\right)=(0, \ldots, 0,1+$ $\left.k-k^{\prime}, 0, \ldots, 0\right)$. We consider the remaining case that $b_{k^{\prime}} \in\left\{0,1, \ldots, k-k^{\prime}\right\}$. Then, there exists an index $k^{*} \in\left\{k^{\prime}-1, k^{\prime}-2 \ldots, 2,1\right\}$ satisfying

$$
\begin{aligned}
& 1+k-k^{\prime} \quad>b_{k^{\prime}}, \\
& 1+k-\left(k^{\prime}-1\right)>b_{k^{\prime}}+b_{k^{\prime}-1}, \\
& \text { : } \\
& 1+k-\left(k^{*}+1\right)>b_{k^{\prime}}+b_{k^{\prime}-1}+\cdots+b_{k^{*}+1}, \\
& 1+k-k^{*} \quad=b_{k^{\prime}}+b_{k^{\prime}-1}+\cdots+b_{k^{*}+1}+b_{k^{*}} \text {, } \\
& 0 \quad=b_{k^{*}-1}=\cdots=b_{1} \text {. }
\end{aligned}
$$

The above inequalities and equalities imply that

$$
\begin{array}{ll}
1+\left(k-b_{k^{\prime}}\right)-k^{\prime} & >0, \\
1+\left(k-b_{k^{\prime}}\right)-\left(k^{\prime}-1\right) & >b_{k^{\prime}-1}, \\
& \vdots \\
1+\left(k-b_{k^{\prime}}\right)-\left(k^{*}+1\right) & >b_{k^{\prime}-1}+\cdots+b_{k^{*}+1}, \\
1+\left(k-b_{k^{\prime}}\right)-k^{*} & =b_{k^{\prime}-1}+\cdots+b_{k^{*}+1}+b_{k^{*}}, \\
0 & =b_{k^{*}-1}=\cdots=b_{1},
\end{array}
$$

and thus we have $\left(0, \ldots, 0,0, b_{k^{\prime}-1}, \ldots, b_{1}\right) \in \Xi_{k-b_{k^{\prime}}}^{0}\left(k^{\prime}-1\right)$. The inverse implication is clear, i.e., $0 \leq \forall b_{k^{\prime}} \leq k-k^{\prime}$, if $\left(0, \ldots, 0,0, b_{k^{\prime}-1}, \ldots, b_{1}\right) \in \Xi_{k-b_{k^{\prime}}}^{0}\left(k^{\prime}-1\right)$, then $\left(0, \ldots, 0, b_{k^{\prime}}, \ldots, b_{1}\right) \in \Xi_{k}^{0}\left(k^{\prime}\right)$. 
From the above, the definition of $\alpha\left(k, k^{\prime}\right)$ implies that

$$
\begin{aligned}
\alpha\left(k, k^{\prime}\right) & =\sum_{\left(0, \ldots, 0, b_{k^{\prime}}, \ldots, b_{1}\right) \in \Xi_{k}^{0}\left(k^{\prime}\right)}\left(\frac{\lambda_{k^{\prime}}^{b_{k^{\prime}}}}{b_{k^{\prime}} !} \frac{\lambda_{k^{\prime}-1}^{b_{k^{\prime}}-1}}{b_{k^{\prime}}-1} \cdots \frac{\lambda_{1}^{b_{1}}}{b_{1} !}\right) \\
& =\sum_{b=0}^{1+k-k^{\prime}} \sum_{\left(0, \ldots, 0, b, b_{k^{\prime}-1}, \ldots, b_{1}\right) \in \Xi_{k}^{0}\left(k^{\prime}\right)}\left(\frac{\lambda_{k^{\prime}}^{b}}{b !} \frac{\lambda_{k^{\prime}-1}^{b_{k^{\prime}}-1}}{b_{k^{\prime}-1} !} \cdots \frac{\lambda_{1}^{b_{1}}}{b_{1} !}\right) \\
& =\frac{\lambda_{k^{\prime}}^{1+k-k^{\prime}}}{\left(1+k-k^{\prime}\right) !}\left(\frac{\lambda_{k^{\prime}-1}^{0}}{0 !} \cdots \frac{\lambda_{1}^{0}}{0 !}\right)+\sum_{b=0}^{k-k^{\prime}}\left(\frac{\lambda_{k^{\prime}}^{b}}{b !} \sum_{\left(0, \ldots, 0,0, b_{k^{\prime}-1}, \ldots, b_{1}\right) \in \Xi_{k-b}^{0}\left(k^{\prime}-1\right)}\left(\frac{\lambda_{k^{\prime}-1}^{b_{k^{\prime}}-1}}{b_{k^{\prime}-1} !} \cdots \frac{\lambda_{1}^{b_{1}}}{b_{1} !}\right)\right) \\
& =\frac{\lambda_{k^{\prime}}^{1+k-k^{\prime}}}{\left(1+k-k^{\prime}\right) !}+\sum_{b=0}^{k-k^{\prime}}\left(\frac{\lambda_{k^{\prime}}^{b}}{b !} \alpha\left(k-b, k^{\prime}-1\right)\right) .
\end{aligned}
$$

When $k=k^{\prime}$, the above equality implies that

$$
1=\alpha\left(k^{\prime}, k^{\prime}\right)=\frac{\lambda_{k^{\prime}}}{1 !}+\frac{\lambda_{k^{\prime}}^{0}}{0 !} \alpha\left(k^{\prime}, k^{\prime}-1\right)=\lambda_{k^{\prime}}+\alpha\left(k^{\prime}, k^{\prime}-1\right)
$$

We assume the following induction hypothesis

$$
1=\alpha\left(k^{\prime}-1, k^{\prime}-1\right)>\alpha\left(k^{\prime}, k^{\prime}-1\right)>\cdots>\alpha\left(m, k^{\prime}-1\right)>0 .
$$

Then, for any integer $k \in\left\{k^{\prime}, k^{\prime}+1, \ldots, m\right\}$, we can show that

$$
\begin{aligned}
& \alpha\left(k, k^{\prime}\right)-\alpha\left(k+1, k^{\prime}\right) \\
& =\frac{\lambda_{k^{\prime}}^{1+k-k^{\prime}}}{\left(1+k-k^{\prime}\right) !}+\sum_{b=0}^{k-k^{\prime}}\left(\frac{\lambda_{k^{\prime}}^{b}}{b !} \alpha\left(k-b, k^{\prime}-1\right)\right)-\frac{\lambda_{k^{\prime}}^{2+k-k^{\prime}}}{\left(2+k-k^{\prime}\right) !}-\sum_{b=0}^{1+k-k^{\prime}}\left(\frac{\lambda_{k^{\prime}}^{b}}{b !} \alpha\left(k+1-b, k^{\prime}-1\right)\right) \\
& =\frac{\lambda_{k^{\prime}}^{1+k-k^{\prime}}}{\left(1+k-k^{\prime}\right) !}-\frac{\lambda_{k^{\prime}}^{2+k-k^{\prime}}}{\left(2+k-k^{\prime}\right) !}-\frac{\lambda_{k^{\prime}}^{1+k-k^{\prime}}}{\left(1+k-k^{\prime}\right) !} \alpha\left(k^{\prime}, k^{\prime}-1\right) \\
& +\sum_{b=0}^{k-k^{\prime}}\left(\frac{\lambda_{k^{\prime}}^{b}}{b !}\left(\alpha\left(k-b, k^{\prime}-1\right)-\alpha\left(k+1-b, k^{\prime}-1\right)\right)\right) \\
& \geq \frac{\lambda_{k^{\prime}}^{1+k-k^{\prime}}}{\left(1+k-k^{\prime}\right) !}\left(1-\alpha\left(k^{\prime}, k^{\prime}-1\right)\right)-\frac{\lambda_{k^{\prime}}^{2+k-k^{\prime}}}{\left(2+k-k^{\prime}\right) !} \quad \text { (obtained from (20)) } \\
& =\frac{\lambda_{k^{\prime}}^{1+k-k^{\prime}}}{\left(1+k-k^{\prime}\right) !} \lambda_{k^{\prime}}-\frac{\lambda_{k^{\prime}}^{2+k-k^{\prime}}}{\left(2+k-k^{\prime}\right) !} \\
& =\frac{\lambda_{k^{\prime}}^{2+k-k^{\prime}}}{\left(1+k-k^{\prime}\right) !}\left(1-\frac{1}{2+k-k^{\prime}}\right)>0 \text {. }
\end{aligned}
$$

Now we describe a proof of Theorem 3.2. 
Proof of Theorem 3.2. Obviously, $\lambda_{1}=1>0$. Equalities (19) and Lemma 5.1 imply that

$\forall k^{\prime} \in\{2,3, \ldots, m\}, \quad \lambda_{k^{\prime}}=1-\alpha\left(k^{\prime}, k^{\prime}-1\right)=\alpha\left(k^{\prime}-1, k^{\prime}-1\right)-\alpha\left(k^{\prime}, k^{\prime}-1\right)>0$.

Recurrence relations (18) and (19) give the following efficient algorithm for calculating $\left(\lambda_{1}, \lambda_{2}, \ldots, \lambda_{m}\right)$ without enumerating vectors in $\Xi_{k}$.

$\underline{\text { Algorithm A }}$

Step 0: Set $k^{\prime}:=1 ; \lambda_{1}:=1 ; \alpha(k, 1):=1 / k$ ! for all $k \in\{1,2, \ldots, m\}$.

Step 1: Set $k^{\prime}:=k^{\prime}+1 ; \alpha\left(k^{\prime}, k^{\prime}\right):=1 ; \lambda_{k^{\prime}}:=1-\alpha\left(k^{\prime}, k^{\prime}-1\right)$.

For each $k \in\left\{k^{\prime}+1, \ldots, m\right\}$, calculate $\alpha\left(k, k^{\prime}\right)$ by (18).

Step 2: If $k^{\prime}=m$, then stop and output $\left(\lambda_{1}, \lambda_{2}, \ldots, \lambda_{m}\right)$. Else, goto Step 1.

The total number of basic arithmetic operations required in Algorithm A is bounded by $\mathrm{O}\left(\mathrm{m}^{3}\right)$.

\section{Acknowledgments.}

The authors thank Professor A. V. Gnedin for his comment on using a Poisson approximation to find the asymptotic probability of win at the 34th Conference on Stochastic Processes and their Applications, Osaka.

This work was supported by JSPS KAKENHI Grant Numbers 26285045, 26242027 .

\section{References}

Ano, K. 2001. Multiple selection problem and OLA stopping rule. Sci. Math. Japon 53(2) 335-346.

Ano, K., N. Kakie, N. Miyoshi. 2011. Odds theorem in Markov-dependent trials with multiple selection chances. RIMS Kokyuroku, Kyoto University 1734 212-219.

Ano, K., H. Kakinuma, N. Miyoshi. 2010. Odds theorem with multiple selection chances. J. Appl. Probab. 47(4) 1093-1104.

Assaf, D., E. Samuel-Cahn. 2000. Simple ratio prophet inequalities for a mortal with multiple choices. J. Appl. Probab. 37(4) 1084-1091.

Bruss, F. T. 1988. Invariant record processes and applications to best choice modelling. Stochastic Process Appl. 30(2) 303-316.

Bruss, F. T. 2000. Sum the odds to one and stop. Ann. Probab. 28(3) 1384-1391. 
Bruss, F. T. 2003. A note on bounds for the odds theorem of optimal stopping. Ann. Probab. 31(4) 1859-1861.

Bruss, F. T., G. Louchard. 2009. The odds algorithm based on sequential updating and its performance. Adv. Appl. Probab. 41(1) 131-153.

Bruss, F. T., D. Paindaveine. 2000. Selecting a sequence of last successes in independent trials. J. Appl. Probab. 37(2) 389-399.

Chow, Y. S., H. Robbins, D. O. Siegmund. 1971. Great expectations : the theory of optimal stopping. Houghton Mifflin, Boston.

Ferguson, T. S. 1989. Who solved the secretary problem? Stat. Sci. 4(3) $282-296$.

Ferguson, T. S. 2006. Optimal stopping and applications. Electronic text available at http://www.math.ucla.edu/tom/Stopping/Contents.html.

Ferguson, T. S. 2008. The sum-the-odds theorem with application to a stopping game of Sakaguchi. Preprint.

Gilbert, J. P., F. Mosteller. 1966. Recognizing the maximum of a sequence. J. Amer. Stat. Assoc. 61 35-73.

Gnedin, A. V. 2010. Private communication.

Hill, T. P., D. P. Kennedy. 1992. Sharp inequalities for optimal stopping with rewards based on ranks. Ann. Appl. Probab. 2(2) 503-517.

Hill, T. P., U. Krengel. 1992. A prophet inequality related to the secretary problem. Contemp. Math. 125 209-215.

Hsiau, S. R., J. R. Yang. 2002. Selecting the last success in Markov-dependent trials. J. Appl. Probab. 39(2) 271-281.

Matsui, M., K. Ano. 2012. Lower Bounds for Bruss' Odds Problem with Multiple Stoppings. arXiv:1204.5537v1 [math.PR].

Pfeifer, D. 1989. Extremal processes, secretary problems and the 1/e-law. J. Appl. Probab. 26(4) 722-733.

Samuels, S. M. 1992. Secretary problems as a source of benchmark bounds. Stochastic inequalities, IMS Lecture Notes Monogr. Ser., vol. 22. Inst. Math. Statist., 371-387.

Shiryaev, A. N. 2008. Optimal stopping rules, Stochastic Modelling and Applied Probability, vol. 8. Springer-Verlag, Berlin. Translated from the 1976 Russian second edition by A. B. Aries, Reprint of the 1978 translation.

Tamaki, M. 2010. Sum the multiplicative odds to one and stop. J. Appl. Probab. 47(3) $761-777$. 